University of Nebraska - Lincoln

DigitalCommons@University of Nebraska - Lincoln

Faculty Publications, Department of Mathematics

Mathematics, Department of

8-19-1997

\title{
INVARIANT SUBSPACES AND HYPER-REFLEXIVITY FOR FREE SEMIGROUP ALGEBRAS
}

Kenneth R. Davidson

University of Waterloo, krdavidson@math.uwaterloo.ca

David R. Pitts

University of Nebraska-Lincoln, dpitts@math.unl.edu

Follow this and additional works at: https://digitalcommons.unl.edu/mathfacpub

Part of the Applied Mathematics Commons, and the Mathematics Commons

Davidson, Kenneth R. and Pitts, David R., "INVARIANT SUBSPACES AND HYPER-REFLEXIVITY FOR FREE SEMIGROUP ALGEBRAS" (1997). Faculty Publications, Department of Mathematics. 242.

https://digitalcommons.unl.edu/mathfacpub/242

This Article is brought to you for free and open access by the Mathematics, Department of at DigitalCommons@University of Nebraska - Lincoln. It has been accepted for inclusion in Faculty Publications, Department of Mathematics by an authorized administrator of DigitalCommons@University of Nebraska - Lincoln. 


\title{
INVARIANT SUBSPACES AND HYPER-REFLEXIVITY FOR FREE SEMIGROUP ALGEBRAS
}

\author{
KENNETH R. DAVIDSON and DAVID R. PITTS
}

[Received 19 August 1997]

In this paper, we obtain a complete description of the invariant subspace structure of an interesting new class of algebras which we call free semigroup algebras. This enables us to prove that they are reflexive, and moreover to obtain a quantitative measure of the distance to these algebras in terms of the invariant subspaces. Such algebras are called hyper-reflexive. This property is very strong, but it has been established in only a very few cases. Moreover the prototypes of this class of algebras are the natural candidate for a non-commutative analytic Toeplitz algebra on $n$ variables. The case we make for this analogy is very compelling. In particular, in this paper, the key to the invariant subspace analysis is a good analogue of the Beurling theorem for invariant subspaces of the unilateral shift. This leads to a notion of inner-outer factorization in these algebras. In a sequel to this paper [13], we add to this evidence by showing that there is a natural homomorphism of the automorphism group onto the group of conformal automorphisms of the ball in $\mathbb{C}^{n}$.

A free semigroup algebra is the weak operator topology closed algebra generated by a set $S_{1}, \ldots, S_{n}$ of isometries with pairwise orthogonal ranges. These conditions are described algebraically by

$$
S_{i}^{*} S_{j}=\delta_{i j} I \quad \text { for } 1 \leqslant i, j \leqslant n,
$$

or equivalently by

$$
S_{i}^{*} S_{i}=I \quad \text { for } 1 \leqslant i \leqslant n \quad \text { and } \quad \sum_{i=1}^{n} S_{i} S_{i}^{*} \leqslant I .
$$

Let $\mathscr{F}_{n}$ denote the unital free semigroup generated by $z_{1}, \ldots, z_{n}$. This semigroup consists of all non-commuting words $w$ in the generators. We are considering those representations of $\mathscr{F}_{n}$ as isometries on Hilbert space determined by such an $n$-tuple of isometries; sending $w$ to $w\left(S_{1}, \ldots, S_{n}\right)$. We allow $n$ to be any positive integer or $\infty$, although for notational convenience we will act as if $n$ is finite. When there is any distinction for the infinite case, it will be noted.

Ordered tuples of isometries satisfying (F) have been studied in various contexts. In $\mathrm{C}^{*}$-algebra theory, they arise as the generators of two important algebras [9], the Cuntz algebras $\mathcal{O}_{n}$ when $\sum_{i} S_{i} S_{i}^{*}=I$ and the Cuntz-Toeplitz extension $\mathscr{E}_{n}$ of $\mathcal{O}_{n}$ by the compact operators when this sum is strictly less than the identity. These algebras have played an important role in the modern $\mathrm{C}^{*}$ theory. The left regular representation of $\mathscr{F}_{n}$ acts on the full Fock space generated

The work of the first author was partially supported by an NSERC grant and a Killam Research Fellowship. That of the second author was partially supported by an NSF grant.

1991 Mathematics Subject Classification: 47D25.

Proc. London Math. Soc. (3) 78 (1999) 401-430. 
by $\mathbb{C}^{n}$. This representation occurs in certain $C^{*}$-algebraic formulations of quantum mechanics (cf. [26, X.7; 6, 5.2]).

Recent work of Bratteli and Jorgensen [5] decomposes certain permutation representations of the Cuntz algebra into irreducible representations in order to study wavelets. It turns out that they consider a class of representations which are a subset of those which we call atomic. We will obtain a complete classification of these representations. This will enable us to describe completely the invariant subspace structure of the atomic free semi-group algebras.

These algebras also arise in dilation theory. Frahzo [14, 15] and Bunce [7] show that any $n$-tuple $T=\left(T_{1}, \ldots, T_{n}\right)$ of operators in $\mathscr{B}(\mathscr{H})$ satisfying $\sum_{i} T_{i} T_{i}^{*} \leqslant I$ may be dilated to an $n$-tuple of isometries satisfying $\left(\mathrm{F}^{\prime}\right)$. This circle of ideas has been elaborated on in a series of papers by Popescu $[19,20,22,24,25]$ generalizing many important results from the Sz. Nagy-Foiaş theory [29] to the multi-variable context.

Of particular interest is the left regular free semigroup algebra $\mathfrak{L}_{n}$, determined by the left regular representation of $\mathscr{F}_{n}$ which acts on $\ell_{2}\left(\mathscr{F}_{n}\right)$ by $\lambda(w) \xi_{v}=\xi_{w v}$ for $v, w$ in $\mathscr{F}_{n}$. The algebra $\mathfrak{L}_{n}$ is generated by the isometries $L_{i}=\lambda\left(z_{i}\right)$ for $1 \leqslant i \leqslant n$. For $n=1$, we obtain the algebra generated by the unilateral shift, the analytic Toeplitz algebra. For $n \geqslant 2$, we obtain a natural non-commutative analogue of the Toeplitz algebra. In particular, there is a reasonable analogue of Beurling's Theorem and inner-outer factorization. There is a plethora of point evaluations for the complex $n$-ball from eigenvalues of the adjoint. However, because of the non-commutative nature, these vectors do not span the whole space; and thus do not provide a complete picture. Nevertheless, the complex ball provides an important connection between this algebra and complex function theory.

Our study begins with a careful look at the left regular free semigroup algebra. We first show that $\mathfrak{R}_{n}$ is the commutant of $\mathfrak{R}_{n}$, the right regular representation algebra. This leads to information about the spectrum of operators of $\mathfrak{L}_{n}$. In particular, every non-scalar element has connected spectrum containing more than one point. These operators are always injective, and thus have no eigenvectors. This in turn leads to the fact that $\mathfrak{L}_{n}$ is inverse closed and semisimple.

We establish a detailed structure of the invariant subspace lattice of $\mathfrak{L}_{n}$ that parallels Beurling's Theorem [4] for the case $n=1$. Every invariant subspace is determined by a wandering space, and can be written as a direct sum of cyclic invariant subspaces. Moreover, akin to the characterization of invariant subspaces of the shift by inner functions, every cyclic invariant subspace is the range of an isometry in the commutant $\mathfrak{R}_{n}$. This leads to the inner-outer factorization: every element $A$ in $\mathfrak{Q}_{n}$ factors as $A=L B$ in $\mathfrak{L}_{n}$, where $L$ is an isometry and $B$ has dense range. Certain invariant subspaces correspond to point evaluations in the complex $n$-ball $\mathbb{B}_{n}=\left\{z \in \mathbb{C}^{n}:\|z\|<1\right\}$. Indeed, for each point $\lambda$ in $\mathbb{B}_{n}$, there is a vector $\nu_{\lambda}$ which is an eigenvector for the adjoint which determines a WOT-continuous multiplicative linear function $\varphi_{\lambda}(A)=\left(A \nu_{\lambda}, \nu_{\lambda}\right)$. These functionals play a pervasive role in our analysis. However, unlike the $n=1$ case, these vectors do not generate the whole lattice because they are symmetric (depending only on the evaluation of words on commuting variables).

An operator algebra $\mathfrak{A}$ is reflexive if the algebra can be recovered from its invariant subspace lattice $\mathscr{L}=\operatorname{Lat}(\mathfrak{U})$ as the set $\operatorname{Alg}(\mathscr{L})$ of all operators leaving 
each subspace invariant. The lattice $\mathscr{L}$ determines a seminorm on $\mathscr{B}(\mathscr{H})$ by

$$
\beta_{\mathscr{L}}(T):=\sup _{L \in \mathscr{L}}\left\|L^{\perp} T L\right\| .
$$

Clearly, $\beta_{\mathscr{L}}(T)=0$ precisely when $T$ belongs to $\operatorname{Alg}(\mathscr{L})$. Moreover, it is elementary to show that

$$
\beta_{\mathscr{L}}(T) \leqslant \operatorname{dist}(T, \operatorname{Alg}(\mathscr{L})) \quad \text { for all } T \in \mathscr{B}(\mathscr{H}) .
$$

The algebra is said to be hyper-reflexive if these norms are comparable. In this case, the constant of hyper-reflexivity is the smallest number $C$ such that

$$
\operatorname{dist}(T, \operatorname{Alg}(\mathscr{L})) \leqslant C \beta_{\mathscr{L}}(T) \quad \text { for all } T \in \mathscr{B}(\mathscr{H}) .
$$

The list of algebras known to be hyper-reflexive is rather short. It includes nest algebras [3] which have constant 1 and injective von Neumann algebras [8] which have constant at most 4 (von Neumann algebras with abelian commutant have constant at most 2, as do abelian von Neumann algebras [27]). And the case most closely related to our study is the analytic Toeplitz algebra $\mathscr{T}\left(H^{\infty}\right)=\mathfrak{L}_{1}$ which has distance constant at most 19 [11].

We will show that $\mathfrak{I}_{n}$ is hyper-reflexive with distance constant at most 51 for $n \geqslant 2$. We also show that this algebra has property $\mathbb{A}_{1}$, meaning that every weak-* continuous linear functional $\varphi$ on $\mathfrak{L}_{n}$ can be represented by a rank 1 functional $\varphi(A)=(A \xi, \zeta)$ such that $\|\xi\|\|\zeta\| \leqslant(1+\varepsilon)\|\varphi\|$ for any positive $\varepsilon$. This leads to the conclusion that every unital WOT-closed subalgebra of $\mathfrak{L}_{n}$ is also hyper-reflexive. Since $\mathfrak{L}_{n}$ is its own double commutant, there is another estimate of the distance to $\mathfrak{L}_{n}$ obtained from the norm of the derivation $\delta_{T}$ restricted to the commutant $\mathfrak{R}_{n}^{\prime}=\mathfrak{R}_{n}$. This seminorm is also shown to be equivalent to the norm distance.

In the last section, we turn our attention to more general free semigroup algebras. Say that a free semigroup algebra is atomic if there is an atomic masa containing the ranges of the isometries $w\left(S_{1}, \ldots, S_{n}\right)$. Equivalently, this means that there is an orthonormal basis $\left\{\xi_{n}\right\}$ for the Hilbert space $\mathscr{H}$, endomorphisms $\pi_{i}$ of $\mathbb{N}$ and scalars $\lambda_{i, n}$ in $\mathbb{T}$ such that $S_{i} \xi_{n}=\lambda_{i, n} \xi_{\pi_{i}(n)}$. These yield an interesting and tractable class of representations of the Cuntz algebra $\mathcal{O}_{n}$ subsuming the class of permutation representations studied in [5]. As $\mathcal{O}_{n}$ is a simple $\mathrm{C}^{*}$-algebra which is not type I, its representations cannot be classified up to unitary equivalence. So it is interesting that this class can be completely classified.

Surprisingly, some of the WOT-closed free semigroup algebras obtained from these representations contain proper projections, which is not the case for $\mathfrak{l}_{n}$. The invariant subspaces can again be classified; and these algebras are shown to be hyper-reflexive.

The key to the analysis is the fact that a basis vector $\xi_{n}$ is either a wandering vector which sweeps out a copy of the left regular representation, or it is fixed by some word $w\left(S_{1}, \ldots, S_{n}\right)$. This word determines an irreducible representation of a special form that has a ring of vectors permuted by those $S_{i}$ occurring, in order, in the word $w$ with $n-1$ copies of the left regular representation coming off each node of the ring. The projection onto this ring lies in the algebra, and every vector in its range is cyclic. The general atomic representation is shown to be a direct sum of representations of three special types: the left regular representation, some doubly infinite variants of the left regular representation, and these ring representations. 
As we were in the process of preparing our results for publication, the paper of Arias and Popescu [1] appeared. Their results overlap somewhat with ours, most notably in $\S 2$ below, however our point of view is somewhat different. They have also pointed out that several of the results we obtained were not new, and appear in previous papers of Popescu, particularly [21, 23]. We wish to thank Arias and Popescu for their comments and we will note the overlaps below.

\section{The left regular free semigroup algebra}

Form a Hilbert space $\mathscr{H}_{n}=\ell_{2}\left(\mathscr{F}_{n}\right)$ with orthonormal basis vectors $\xi_{w}$ for each word $w$ in the unital semigroup $\mathscr{F}_{n}$. Define operators $L_{i}=\lambda\left(z_{i}\right)$ by $L_{i} \xi_{w}=\xi_{z_{i} w}$. It is immediately evident that each $L_{i}$ is an isometry, and that the ranges are pairwise orthogonal. Indeed,

$$
I-\sum_{i=1}^{n} L_{i} L_{i}^{*}=\xi_{1} \xi_{1}^{*}
$$

is a rank 1 projection. Let $\mathfrak{I}_{n}$ denote the unital WOT-closed algebra generated by $L_{1}, \ldots, L_{n}$. It is then evident that $v\left(L_{1}, \ldots, L_{n}\right) \xi_{w}=\xi_{v w}$ for all words $v$ and $w$ in $\mathscr{F}_{n}$. For convenience of notation, we will write $L=\left(L_{1}, \ldots, L_{n}\right)$ and $L_{v}$ or $v(L)$ will denote the corresponding word in the $n$-tuple.

Similarly, the right regular representation is defined by $\rho(w)=R_{\widetilde{w}}$ where

$$
R_{v} \xi_{w}=\xi_{w v} \quad \text { for } v, w \in \mathscr{F}_{n}
$$

and $\widetilde{w}$ denotes the word $w$ in reverse order. Let $R_{i}:=R_{z_{i}}=\rho\left(z_{i}\right)$ denote the images of the generators for $1 \leqslant i \leqslant n$; and let $\mathfrak{R}_{n}$ denote the wOT-closed algebra generated by the $R_{i}$. Denote by $W$ the unitary which sends $\xi_{w}$ to $\xi_{\widetilde{w}}$. It is easily seen that $W L_{i} W^{*}=R_{i}$. Thus $\mathfrak{R}_{n}$ is unitarily equivalent to $\mathfrak{L}_{n}, \rho=\operatorname{Ad}(W) \lambda$, and $W L_{w} W^{*}=R_{\widetilde{w}}$. Notice that $\mathfrak{I}_{n}$ and $\mathfrak{R}_{n}$ commute with each other. It follows that the range projection $P_{v}=R_{v} R_{v}^{*}$ of each right shift is invariant for $\mathfrak{L}_{n}$.

The full Fock space of a Hilbert space $\mathscr{H}$ is the Hilbert space

$$
F(\mathscr{H})=\sum_{k \geqslant 0}^{\oplus} \mathscr{H}^{\otimes k}
$$

where $\mathscr{H}^{\otimes 0}=\mathbb{C}$ and $\mathscr{H}^{\otimes k}$ is the tensor product of $k$ copies of $\mathscr{H}$. When $\mathscr{H}=\mathbb{C}^{n}$ with orthonormal basis $\zeta_{i}$ for $1 \leqslant i \leqslant n$, the Fock space has an orthonormal basis $\zeta_{w}=\zeta_{i_{1}} \otimes \ldots \otimes \zeta_{i_{k}}$ for all choices of $w=\left(i_{1}, \ldots, i_{k}\right)$ in $\{1, \ldots, n\}^{k}$ and $k \geqslant 0$ (with the convention that $\zeta_{\emptyset}$ spans $\mathscr{H}^{\otimes 0}$ ). For each vector $\zeta$ in $\mathscr{H}$, there is a left creation operator $\ell(\zeta) \xi=\zeta \otimes \xi$. Clearly, there is a natural isomorphism of Fock space onto $\mathscr{H}_{n}$, where $n=\operatorname{dim} \mathscr{H}$, given by sending $\zeta_{w}$ to $\xi_{w}$. This unitary equivalence sends $\ell\left(\zeta_{i}\right)$ to $L_{i}$.

The following heuristic is useful when working with operators in $\mathfrak{L}_{n}$. If $A=\sum_{w} a_{w} L_{w}$ is a finite linear combination of the set $\left\{L_{w}: w \in \mathscr{F}_{n}\right\}$, then $A \xi_{1}=\sum_{w} a_{w} \xi_{w}$; conversely, given a finite linear combination of basis vectors $\zeta=\sum_{w} a_{w} \xi_{w}$, the operator $A=\sum_{w} a_{w} L_{w}$ belongs to $\mathfrak{L}_{n}$ and satisfies $A \xi_{1}=\zeta$. This correspondence of course cannot be extended to infinite combinations. However we note that for an arbitrary element $A$ of $\mathfrak{L}_{n}, A$ is completely determined by what it does on $\xi_{1}$ : indeed, $A \xi_{v}=A R_{v} \xi_{1}=R_{v} A \xi_{1}$. So if $A \xi_{1}=\sum_{w} a_{w} \xi_{w}$, we have $A \xi_{v}=\sum a_{w} \xi_{w v}=\sum_{w} a_{w}\left(L_{w} \xi_{v}\right)$. Thus it is sometimes 
useful to view $A$ as the formal sum $\sum_{w} a_{w} L_{w}$ which serves as a Fourier expansion of $A$.

As in the case with classical harmonic analysis, we shall use Cesàro sums to enable us to make sense of such formal sums. Let $Q_{k}$ denote the projection onto $\operatorname{span}\left\{\xi_{w}:|w|=k\right\}$. Let $\Phi_{j}$ denote the completely contractive projections on $\mathscr{B}(\mathscr{H})$ given by

$$
\Phi_{j}(T)=\sum_{k \geqslant \max \{0,-j\}} Q_{k} T Q_{k+j} .
$$

The proof of the following lemma follows from the standard estimates for Fejér's Theorem.

Lemma 1.1. The Cesaro operators on $\mathscr{B}\left(\mathscr{H}_{n}\right)$ defined by

$$
\Sigma_{k}(T)=\sum_{|j|<k}\left(1-\frac{|j|}{k}\right) \Phi_{j}(T) \quad \text { for } k \geqslant 1
$$

are completely contractive. Moreover, for each $T$ in $\mathscr{B}(\mathscr{H})$, the sequence $\Sigma_{k}(T)$ converges to $T$ in the strong operator topology.

Our first result is a direct analogue of the characterizations of the analytic Toeplitz algebra, the $n=1$ case, where $\mathfrak{L}_{1}=\mathfrak{R}_{1}=\mathscr{T}\left(H^{\infty}\right)$.

We note that Theorem 1.2 and Corollary 1.3 also appear in [23] (see Theorem 1.2 and the material following it on page 35 of [23]). However, our proof is quite different.

THEOREM 1.2. For $A$ in $\mathscr{B}\left(\mathscr{H}_{n}\right)$, the following are equivalent:

(i) A belongs to $\mathfrak{L}_{n}$;

(ii) A belongs to $\mathfrak{R}_{n}^{\prime}$;

(iii) $A=R_{v}^{*} A R_{v}$ and $P_{v}$ belongs to $\operatorname{Lat}(\mathrm{A})$ for all $v$ in $\mathscr{F}_{n}$.

Proof. It is clear that (i) implies (ii), and (ii) implies (iii). Suppose that (iii) holds. Then

$$
A R_{v} \xi_{w}=P_{v} A R_{v} \xi_{w}=R_{v}\left(R_{v}^{*} A R_{v}\right) \xi_{w}=R_{v} A \xi_{w}
$$

for all $v, w$ in $\mathscr{F}_{n}$. So (ii) holds.

To show that (ii) implies (i), fix $A$ in $\mathfrak{R}_{n}^{\prime}$ and let $A \xi_{1}=\sum_{w} a_{w} \xi_{w}$. Recall that $|w|$ denotes the length of a word $w$. Consider the Cesàro sums

$$
p_{k}(L)=\sum_{|w|<k}\left(1-\frac{|w|}{k}\right) a_{w} L_{w} .
$$

It is evident that $p_{k}(L)$ belong to $\mathfrak{R}_{n}$. We will show that $p_{k}(L)$ converges wot to $A$.

Note that $\Phi_{j}(A)$ also lies in $\Re_{n}^{\prime}$ because $R_{i} Q_{k}=Q_{k+1} R_{i}$; and thus

$$
\begin{aligned}
R_{i} \Phi_{j}(A) & =\sum_{k} R_{i} Q_{k} A Q_{k+j}=\sum_{k} Q_{k+1} R_{i} A Q_{k+j} \\
& =\sum_{k} Q_{k+1} A Q_{k+j+1} R_{i}=\Phi_{j}(A) R_{i} .
\end{aligned}
$$


Hence $\Sigma_{k}(A)$ also lies in $\Re_{n}^{\prime}$ and converges sot to $A$. Notice that

$$
\Sigma_{k}(A) \xi_{1}=\sum_{|w|<k}\left(1-\frac{|w|}{k}\right) a_{w} \xi_{w}=p_{k}(L) \xi_{1} .
$$

By the remarks preceding Lemma 1.1 , we conclude that $\Sigma_{k}(A)=p_{k}(L)$ belongs to $\mathfrak{Q}_{n}$; and so $A$ does also.

By symmetry, it is also the case that $\mathfrak{R}_{n}=\mathfrak{Q}_{n}^{\prime}$. Thus we have the following.

COROLlaRY 1.3. The algebra $\mathfrak{Q}_{n}$ is its own double commutant $\mathfrak{Q}_{n}=\mathfrak{Q}_{n}^{\prime \prime}$.

Another automatic consequence of being a commutant is as follows.

COROLlaRy 1.4. The algebra $\mathfrak{L}_{n}$ is inverse closed.

We note that Corollary 1.4 also follows from Corollary 3.2 of [23].

Proof. This result holds for any algebra which is a commutant $\mathfrak{R}^{\prime}$ of some algebra $\mathfrak{R}$. Suppose that $A$ in $\mathfrak{R}^{\prime}$ is invertible in $\mathscr{B}(\mathscr{H})$. Then for any $R$ in $\mathfrak{R}$,

$$
A^{-1} R=A^{-1} R A A^{-1}=A^{-1} A R A^{-1}=R A^{-1} .
$$

Hence $A^{-1}$ belongs to $\Re^{\prime}$.

Now we develop some properties of the individual elements of $\mathfrak{L}_{n}$. Some follow easily from the commutant theorem; while the detailed spectral picture requires additional work.

COROLlaRY 1.5. The only normal elements in $\mathfrak{Q}_{n}$ are scalars.

Proof. If $N$ is a normal element of $\mathfrak{Q}_{n}$, define $\alpha=\left(N \xi_{1}, \xi_{1}\right)$. As $\xi_{1}$ is an eigenvector for $\mathfrak{Q}_{n}^{*}$, it follows that $N^{*} \xi_{1}=\bar{\alpha} \xi_{1}$. By normality, $N \xi_{1}=\alpha \xi_{1}$. Since $N$ commutes with $\mathfrak{R}_{n}$, we obtain

$$
N \xi_{w}=N R_{w} \xi_{1}=R_{w} N \xi_{1}=\alpha \xi_{w} .
$$

Hence $N=\alpha I$.

Corollary 1.6. For all $A$ in $\mathfrak{Q}_{n}$, $\|A\|=\|A\|_{e}$. In particular, there are no non-zero compact operators in $\mathfrak{Q}_{n}$.

Proof. For any vector $\xi$ and word $w$ in $\mathscr{F}_{n}$,

$$
\left\|A R_{w} \xi\right\|=\left\|R_{w} A \xi\right\|=\|A \xi\| .
$$

Since $R_{w} \xi$ tends weakly to 0 as $|w|$ tends to infinity for every vector $\xi$ in $\mathscr{H}$, it follows that $\|A\|=\|A\|_{e}$.

Our analysis of the spectrum of elements of $\mathfrak{R}_{n}$ is quite direct. As with the proof of the commutant theorem, it relies on the fact that each element $A$ of $\mathfrak{L}_{n}$ is uniquely determined by $A \xi_{1}$, which has a 'Fourier expansion' in the standard basis. It also makes use of certain evident invariant subspaces arising from this standard basis. 
THEOREM 1.7. Every non-zero element of $\mathfrak{L}_{n}$ is injective and has non-zero spectrum. For $n \geqslant 2$ and all $A$ in $\mathfrak{L}_{n}, \sigma(A)=\sigma_{e}(A)$.

Proof. Let $A$ in $\mathfrak{L}_{n}$ be a non-zero element; and let $A \xi_{1}=\sum_{w} a_{w} \xi_{w}$. This is non-zero, for otherwise $A \xi_{w}=R_{w} A \xi_{1}=0$ for all $w$, whence $A=0$. Choose a word $v$ of minimal length such that $a_{v} \neq 0$. Then for any basis vector $\xi_{u}$,

$$
A \xi_{u}=R_{u} A \xi_{1}=\sum_{w} a_{w} \xi_{w u}
$$

Thus the non-zero terms correspond to words of length at least $|u|+|v|$. Therefore it follows recursively that

Therefore

$$
A^{k} \xi_{1}=a_{v}^{k} \xi_{v^{k}}+\sum_{w \neq v^{k},|w| \geqslant|v|^{k}} a_{w, k} \xi_{w} .
$$

$$
\left\|A^{k}\right\|^{1 / k} \geqslant\left|\left(A^{k} \xi_{1}, \xi_{v^{k}}\right)\right|^{1 / k}=\left|a_{v}\right| .
$$

So the spectral radius of $A$ is at least $\left|a_{v}\right|>0$.

Now suppose that $\zeta=\sum_{w} b_{w} \xi_{w}$ is a non-zero vector, and let $u$ be a word of minimal length such that $b_{u} \neq 0$. Then computing as above, one sees that

$$
\left(A \zeta, \xi_{v u}\right)=a_{v} b_{u} \neq 0 \text {. }
$$

Therefore $A$ is injective.

Note that to show that $\sigma(A) \subseteq \sigma_{e}(A)$ for every $A$ in $\mathfrak{L}_{n}$, it suffices to show that whenever $A$ belongs to $\mathfrak{I}_{n}$ and 0 belongs to $\sigma(A)$, then 0 lies in $\sigma_{e}(A)$. So suppose 0 belongs to $\sigma(A)$. By Corollary 1.4 and the fact that non-zero elements of $\mathfrak{L}_{n}$ are injective, we see that the only way this can happen is if $A$ is not surjective. If $\operatorname{Ran}(A)$ is not closed, then $A$ is not Fredholm, so 0 is in $\sigma_{e}(A)$.

Suppose then that 0 belongs to $\sigma(A)$ because $\operatorname{Ran}(A)$ is closed and proper. The range of $A$ is $\Re_{n}$ invariant. Thus it does not contain $\xi_{1}$, as this is cyclic for $\Re_{n}$. We claim that

$$
\left(A \operatorname{span}\left\{\xi_{w}:|w| \geqslant k\right\}\right) \cap \operatorname{span}\left\{\xi_{w}:|w| \leqslant k\right\}=\{0\} .
$$

Indeed, $\operatorname{span}\left\{\xi_{w}:|w| \geqslant k\right\}=\sum_{|w|=k}^{\oplus} R_{w} \mathscr{H}_{n}$ is invariant for $\mathfrak{L}_{n}$. Thus the only possible vectors in the intersection have the form

$$
\xi=\sum_{|w|=k} a_{w} \xi_{w}=A \sum_{|w|=k} R_{w} \zeta_{w}
$$

for some vectors $\zeta_{w}$ in $\mathscr{H}_{n}$. But if $|v|=k$, then

$$
a_{v} \xi_{1}=R_{v}^{*} \xi=\sum_{|w|=k} R_{v}^{*} R_{w} A \zeta_{w}=A \zeta_{v} .
$$

Hence $a_{v}=0$ for all $v$; whence $\xi=0$. So (1) holds.

Therefore,

$$
\begin{aligned}
\operatorname{dim}\left(\operatorname{Ran}(A) \cap \operatorname{span}\left\{\xi_{w}:|w| \leqslant k\right\}\right) & \leqslant \operatorname{dim} \operatorname{span}\left\{\xi_{w}:|w|<k\right\} \\
& =\operatorname{dim} \operatorname{span}\left\{\xi_{w}:|w| \leqslant k\right\}-n^{k} .
\end{aligned}
$$

Hence $\operatorname{Ran}(A)$ has codimension at least $n^{k}$ for all $k \geqslant 0$.

Therefore, for $2 \leqslant n<\infty$, this shows that $\operatorname{Ran}(A)$ has infinite codimension. 
When $n=\infty$, the argument still shows that

$$
\operatorname{Ran}(A) \cap \operatorname{span}\left\{\xi_{w}:|w|=1\right\}
$$

is at most 1-dimensional with the same conclusion. Thus 0 belongs to $\sigma_{e}(A)$.

COROLlaRY 1.8. The algebra $\mathfrak{L}_{n}$ contains no non-trivial idempotents or non-zero quasinilpotent elements. Thus the spectrum of every non-scalar element is connected and contains more than one point.

Proof. A non-trivial idempotent has kernel, and a non-zero quasinilpotent element has zero spectrum, both contradicting Theorem 1.7. The Riesz functional calculus provides a proper idempotent in the algebra generated by any operator with disconnected spectrum. Thus the spectrum of every element of $\mathfrak{L}_{n}$ is connected. If $\sigma(A)=\{\lambda\}$ is a singleton, then $A-\lambda I$ is quasinilpotent and therefore equals 0 . So $A$ is scalar.

This yields the following important algebraic consequence.

COROLlary 1.9. The algebra $\mathfrak{Q}_{n}$ is semisimple.

Proof. The radical is contained in the set of quasinilpotent elements, and therefore is $\{0\}$.

REMARK 1.10. During recent correspondence with Arias, we learned that Katsoulis in 1994 also observed that $\mathfrak{I}_{n}$ is semisimple, but we do not have a reference.

We include a computational lemma which will be useful later.

LEMMA 1.11. If $A$ belongs to $\mathfrak{I}_{n}$, then $R_{j}^{*} A-A R_{j}^{*}=\left(R_{j}^{*} A \xi_{1}\right) \xi_{1}^{*}$ is a rank 1 operator. Therefore every element of the norm closed algebra generated by $C^{*}\left(R_{1}, \ldots, R_{n}\right)+\mathfrak{R}_{n}$ commutes with $\mathfrak{R}_{n}$ modulo the compact operators.

Proof. This is a straight-forward computation:

$$
\begin{aligned}
R_{j}^{*} A-A R_{j}^{*} & =R_{j}^{*} A\left(\xi_{1} \xi_{1}^{*}+\sum_{i=1}^{n} R_{i} R_{i}^{*}\right)-A R_{j}^{*} \\
& =\left(R_{j}^{*} A \xi_{1}\right) \xi_{1}^{*}+\sum_{i=1}^{n} R_{j}^{*} R_{i} A R_{i}^{*}-A R_{j}^{*}=\left(R_{j}^{*} A \xi_{1}\right) \xi_{1}^{*} .
\end{aligned}
$$

Since each $R_{j}$ and $R_{j}^{*}$ commutes with $\mathfrak{Q}_{n}$ modulo the compact operators, as does $\mathfrak{R}_{n}$, this fact extends to the norm-closed algebra that they generate.

It would be interesting to know if the converse is true. This is the case for $n=1$, where $C^{*}\left(R_{1}\right)+\Re_{1}=\mathscr{T}\left(H^{\infty}+C\right)$ is known to be the essential commutant of the Toeplitz algebra [10]. For $n \geqslant 2, C^{*}\left(R_{1}, \ldots, R_{n}\right)+\mathfrak{R}_{n}$ is still closed but is no longer an algebra. The fact that it is closed can be established by mimicking the second proof given in [28]. One uses a Cesàro mean argument to establish that

$$
\operatorname{dist}\left(X, \mathscr{A}_{n}\right)=\operatorname{dist}\left(X, \Re_{n}\right) \quad \text { for all } X \in C^{*}\left(R_{1}, \ldots, R_{n}\right),
$$


where $\mathscr{A}_{n}$ is the norm closed algebra generated by $R$. Indeed, $X$ is the norm limit $\lim _{k \rightarrow \infty} \Sigma_{k}(X)$. So if $A$ in $\Re_{n}$ satisfies $\|X-A\|=\operatorname{dist}\left(X, \Re_{n}\right)$, then

$$
\begin{aligned}
\operatorname{dist}\left(X, \mathscr{A}_{n}\right) & \leqslant \lim _{k \rightarrow \infty}\left\|X-\Sigma_{k}(A)\right\| \\
& \leqslant \lim _{k \rightarrow \infty}\left\|X-\Sigma_{k}(X)\right\|+\left\|\Sigma_{k}(X-A)\right\|=\operatorname{dist}\left(X, \Re_{n}\right) .
\end{aligned}
$$

Hence the injection

$$
C^{*}\left(R_{1}, \ldots, R_{n}\right) / \mathscr{A}_{n} \hookrightarrow \mathscr{B}\left(\mathscr{H}_{n}\right) / \Re_{n}
$$

is isometric, and thus has closed range. Pulling this back to $\mathscr{B}\left(\mathscr{H}_{n}\right)$ shows that $C^{*}\left(R_{1}, \ldots, R_{n}\right)+\mathfrak{R}_{n}$ is closed. Using a result on the structure of finitely generated ideals in $\mathfrak{Q}_{n}$ from [13], one can show that finite sums of the form $\sum A_{w} R_{w}^{*}$ with coefficients $A_{w}$ in $\Re_{n}$ are dense in the algebra this generates.

\section{Invariant subspaces for $\mathfrak{L}_{n}$}

Following Popescu's version of the Wold decomposition [19], say that a subspace $\mathscr{W}$ is wandering for an $n$-tuple of isometries $S=\left(S_{1}, \ldots, S_{n}\right)$ satisfying $\left(\mathrm{F}^{\prime}\right)$ if $w(S) \mathscr{W}$ are pairwise orthogonal for distinct words $w$ in $\mathscr{F}_{n}$. Clearly, every wandering subspace generates the invariant subspace $\mathfrak{A}[\mathscr{W}]=\sum_{w}^{\oplus} w(S) \mathscr{W}$ for $\mathfrak{A}=\operatorname{Alg}\left\{S_{1}, \ldots, S_{n}\right\}$. When $\mathscr{W}$ is spanned by a single unit vector $\zeta$, we shall also use the notation $\mathfrak{A}[\zeta]$ for the cyclic subspace generated by $\zeta$. The subspaces $\mathfrak{A}[\mathscr{W}]$ are easily seen to be a direct sum of cyclic subspaces. Indeed, let $\left\{\zeta_{j}\right\}$ denote an orthonormal basis for $\mathscr{W}$. Then $\mathfrak{A}\left[\zeta_{j}\right]$ are pairwise orthogonal $\mathfrak{A}$-invariant subspaces which sum to $\mathfrak{A}[\mathscr{W}]$. Popescu shows that every $\mathfrak{A}$-invariant subspace decomposes as the direct sum of an $\mathfrak{A}[\mathscr{W}]$ space and an invariant subspace $\mathscr{M}$ such that $T_{i}=\left.S_{i}\right|_{\mathscr{M}}$ satisfy

$$
\sum_{i=1}^{n} T_{i} T_{i}^{*}=I .
$$

Unlike the $n=1$ case, this latter condition does not yield much information. When we study other free semigroup algebras, a more delicate analysis will be required.

In the case of the left regular representation, all invariant subspaces are generated by a wandering subspace. This result should be seen as the analogue of Beurling's Theorem for the analytic Toeplitz algebra. In the $n=1$ case, every invariant subspace is cyclic of the form $\omega H^{2}$ where $\omega$ is an inner function. These subspaces are therefore the range of the isometries $T_{\omega}$, which are all the isometries in $\mathscr{T}\left(H^{\infty}\right)=\mathfrak{L}_{1}=\mathfrak{R}_{1}$. The following result has a significant overlap with Theorem 2.3 of [1].

THEOREM 2.1. Every invariant subspace of $\mathfrak{Q}_{n}$ is generated by a wandering subspace. Thus it is the direct sum of cyclic subspaces. The cyclic invariant subspaces of $\mathfrak{L}_{n}$ are precisely the ranges of isometries in $\mathfrak{R}_{n}$; and the choice of isometry is unique up to a scalar.

Proof. Given an invariant subspace $\mathscr{M}$, form the subspace

$$
\mathscr{W}=\mathscr{M} \ominus\left(\sum_{i=1}^{n}{ }^{\oplus} L_{i} \mathscr{M}\right) .
$$


It is clear that $\mathscr{W}$ is orthogonal to $L_{w} \mathscr{W}$ for all $w \neq 1$; and hence it is easy to see that the $L_{w} \mathscr{W}$ are pairwise orthogonal. So $\mathscr{W}$ is wandering. It remains to show that $\mathscr{M}=\mathfrak{L}_{n}[\mathscr{W}]$. By the Wold decomposition, the complement $\mathscr{N}$ of $\mathfrak{L}_{n}[\mathscr{W}]$ in $\mathscr{M}$ is invariant for $\mathfrak{L}_{n}$ and $\mathscr{N}=\sum_{i=1}^{n} L_{i} \mathscr{N}$. If $\mathscr{N}$ is non-zero, choose the smallest integer $k_{0}$ such that $Q_{k_{0}} \mathscr{N} \neq 0$. (Recall that $Q_{k_{0}}$ is the projection onto $\operatorname{span}\left\{\xi_{w}:|w|=k_{0}\right\}$.) But then

$$
Q_{k_{0}} \mathscr{N} \subseteq \sum_{i=1}^{n} Q_{k_{0}} L_{i} \mathscr{N}=\sum_{i=1}^{n} L_{i} Q_{k_{0}-1} \mathscr{N}=0 .
$$

This contradiction shows that $\mathscr{M}=\mathfrak{Q}_{n}[\mathscr{W}]$ as desired.

Now $\mathscr{M}$ is cyclic precisely when $\mathscr{W}$ is 1 -dimensional. Consider a cyclic invariant subspace $\mathfrak{L}_{n}[\zeta]$ where $\zeta$ is a unit wandering vector. Define an isometry $R_{\zeta}$ by the rule

$$
R_{\zeta} \xi_{w}=L_{w} \zeta \quad \text { for } w \in \mathscr{F}_{n}
$$

It is evident by construction that $R_{\zeta}$ commutes with the operators $L_{w}$. So $R_{\zeta}$ belongs to $\mathfrak{R}_{n}$ by Theorem 1.2. Moreover, the range of $R_{\zeta}$ is equal to $\mathfrak{I}_{n}[\zeta]$ by design.

Conversely, if $R$ is an isometry in $\mathfrak{R}_{n}$, the range of $R$ is invariant for $\mathfrak{L}_{n}$. Moreover, $\zeta=R \xi_{1}$ is a wandering vector for this range and $R=R_{\zeta}$. So it is cyclic. Finally, suppose that $R^{\prime}$ is another isometry in $\Re_{n}$ with the same range. Then both $\zeta$ and $\zeta^{\prime}=R^{\prime} \xi_{1}$ are wandering vectors for $R \mathscr{H}_{n}$. Therefore they both lie in the 1 -dimensional wandering space $\mathbb{C} \zeta$. Hence $\zeta^{\prime}=\lambda \zeta$ and $R^{\prime}=\lambda R$ for some scalar $\lambda$ in $\mathbb{T}$.

Another classical result of function theory is that every function $f$ in $H^{1}$ has an inner-outer factorization $f=\omega h$. This factorization is useful both in $H^{\infty}$ and in $H^{2}$. We have already noted that the inner functions in $H^{\infty}$ correspond to those analytic Toeplitz operators which are isometries. Thus we define the inner elements of $\mathfrak{I}_{n}$ to be the isometries. It is the case that $h$ in $H^{\infty}$ is outer if and only if $T_{h}$ has dense range. So we define outer elements of $\mathfrak{L}_{n}$ to be those with dense range. This allows us to obtain a natural inner-outer factorization in $\mathfrak{L}_{n}$. Our definition of outer elements differs from the definition which appears in [21]. However, it is not difficult to show that the definitions coincide for operators in $\mathfrak{I}_{n}$. Thus the following result is an immediate consequence of Theorem 4.2 of [21]. We include it for completeness of exposition and to provide a somewhat different proof.

Corollary 2.2. Every $A$ in $\mathfrak{Q}_{n}$ factors as $A=L_{\zeta} B$ where $L_{\zeta}$ is an isometry in $\mathfrak{L}_{n}$ and $B$ belongs to $\mathfrak{Q}_{n}$ and has dense range. This factorization is unique up to $a$ scalar. The operator $B$ is invertible if and only if $A$ has closed range.

Proof. The closed range $\mathscr{M}=\overline{\operatorname{Ran}(A)}=\overline{A \mathfrak{R}_{n} \xi_{1}}=\mathfrak{R}_{n}\left[A \xi_{1}\right]$ is a cyclic invariant subspace for $\mathfrak{R}_{n}$. Therefore, it has a unit wandering vector $\zeta$, which is unique up to a scalar, such that $\mathscr{M}=L_{\zeta} \mathscr{H}_{n}$. Let $B=L_{\zeta}^{*} A$. Evidently, $A=L_{\zeta} B$, and $B$ has dense range. To verify that $B$ belongs to $\mathfrak{L}_{n}$, we show that it commutes with each $R_{j}$. Indeed, by Lemma 1.11 ,

$$
R_{j} B-B R_{j}=\left(R_{j} L_{\zeta}^{*}-L_{\zeta}^{*} R_{j}\right) A=-\xi_{1}\left(R_{j}^{*} L_{\zeta} \xi_{1}\right)^{*} A=-\xi_{1}\left(A^{*} R_{j}^{*} \zeta\right)^{*} .
$$

Recall that $\zeta$ lies in $\mathscr{M} \ominus \sum_{i=1}^{n} R_{i} \mathscr{M}$. Hence $R_{j}^{*} \zeta$ is orthogonal to the subspace 
$\mathscr{M}=\overline{\operatorname{Ran}(A)}$, so $R_{j}^{*} \zeta$ belongs to $\operatorname{ker} A^{*}$, and therefore $A^{*} R_{j}^{*} \zeta=0$. Thus $R_{j} B-B R_{j}=0$ as claimed.

Uniqueness follows from the uniqueness of $\zeta$ up to a scalar. If $A$ has closed range, then $B$ is surjective. By Theorem 1.7, $B$ is injective and thus is invertible in $\mathfrak{L}_{n}$ by Corollary 1.4 . The converse is clear.

Another of Beurling's results is that the cyclic vectors of the shift are precisely the outer functions in $H^{2}$. So if we take this as our notion of an outer function in $\mathscr{H}_{n}$, we obtain an analogue of inner-outer factorization in Fock space. However, we are missing some sort of intrinsic characterization of cyclic vectors and wandering vectors for $\mathfrak{L}_{n}$. This version of inner-outer factorization in $\mathscr{H}_{n}$ is not as satisfying as the algebra version. Compare the following result with Theorem 2.1 of [1].

COROLlaRY 2.3. Every vector $\eta$ in $\mathscr{H}_{n}$ factors as $\eta=R_{\zeta} \xi$ where $\zeta$ is a wandering vector and $\xi$ is a cyclic vector for $\mathfrak{L}_{n}$. This factorization is unique up to a scalar of modulus 1 .

Proof. The cyclic subspace $\mathfrak{I}_{n}[\eta]$ has a wandering vector $\zeta$. Since $R_{\zeta}$ is an isometry in $\mathfrak{L}_{n}^{\prime}$ onto $\mathfrak{L}_{n}[\eta]$, it follows that $\xi=R_{\zeta}^{*} \eta$ is cyclic and $\eta=R_{\zeta} \xi$. The uniqueness up to a scalar follows from the uniqueness of $\zeta$ up to a scalar.

To complete the picture of inner and outer functions, we provide a more intrinsic characterization of these elements. The characterization for inner operators is analogous to the characterization in $H^{\infty}$ that $h$ is inner if and only if $\|h\|_{\infty}=\|h\|_{2}=1$.

Proposition 2.4. An element $A$ in $\mathfrak{Q}_{n}$ is inner (that is, an isometry) if and only if $\|A\|=\left\|A \xi_{1}\right\|=1$.

Proof. If $A$ is an isometry, it is clear that $\|A\|=\left\|A \xi_{1}\right\|=1$. Conversely, if $\left\|A \xi_{1}\right\|=1$, then $A \xi_{w}=R_{w} A \xi_{1}$ are unit vectors for all $w$ in $\mathscr{F}_{n}$. Since $\|A\|=1$, it follows that these vectors are pairwise orthogonal. Hence $A$ is an isometry.

The characterization of outer functions is parallel to the fact that among all $H^{\infty}$-functions with a given absolute value on the boundary, outer functions have the greatest absolute value at the origin.

Proposition 2.5. An element $A$ in $\mathfrak{L}_{n}$ is outer if and only if $\left|\varphi_{0}(A)\right|=\left|\left(A \xi_{1}, \xi_{1}\right)\right|$ is maximal among

$$
\left\{\left|\varphi_{0}(B)\right|: B \in \mathfrak{L}_{n}, B^{*} B=A^{*} A\right\} .
$$

Two outer functions with the same absolute value differ by a multiple of modulus 1.

Before beginning the proof, we note that this result is part of Theorem 2.5 of [23].

Proof. Suppose that $A$ is outer and that $B$ in $\mathfrak{L}_{n}$ satisfies $B^{*} B=A^{*} A$. Then since $A$ has dense range, there is an isometry $L$ in $\mathscr{B}\left(\mathscr{H}_{n}\right)$ such that $B=L A$. 
Consequently, for $1 \leqslant i \leqslant n$,

$$
0=R_{i} B-B R_{i}=\left(R_{i} L-L R_{i}\right) A+L\left(R_{i} A-A R_{i}\right)=\left(R_{i} L-L R_{i}\right) A .
$$

Since $A$ has dense range, it follows that $L$ commutes with each $R_{i}$; and therefore belongs to $\mathfrak{L}_{n}$ by Theorem 1.2. Since $\varphi_{0}$ is multiplicative and contractive on $\mathfrak{I}_{n}$,

$$
\left|\varphi_{0}(B)\right|=\left|\varphi_{0}(L) \varphi_{0}(A)\right| \leqslant\left|\varphi_{0}(A)\right| .
$$

Conversely, this inequality is strict unless $L \xi_{1}=\lambda \xi_{1}$ for some scalar $\lambda$ of modulus 1. But this clearly implies that $L=\lambda I$ and $B=\lambda A$. So if $B$ is not outer, it does not achieve this maximum.

For the analytic Toeplitz algebra, the eigenvalues of the adjoint algebra correspond to point evaluations in the unit disk. For each $\lambda$ in $\mathbb{D}$, there is a unit vector

$$
k_{\lambda}=\left(1-|\lambda|^{2}\right)^{1 / 2}(1-\bar{\lambda} z)^{-1}=\left(1-|\lambda|^{2}\right)^{1 / 2} \sum_{k \geqslant 0}(\bar{\lambda} z)^{k}
$$

such that $T_{z}^{*} k_{\lambda}=\bar{\lambda} k_{\lambda}$. Moreover

$$
\left(T_{h} k_{\lambda}, k_{\lambda}\right)=h(\lambda) \text { for all } h \in H^{\infty} .
$$

Their orthogonal complements are given by $\left\{k_{\lambda}\right\}^{\perp}=b_{\bar{\lambda}} H^{2}$ where

$$
b_{\lambda}(z)=\frac{z-\lambda}{1-\bar{\lambda} z}
$$

is a Mobius map of the unit disk. The lattice generated by these subspaces contains $b H^{2}$ for every Blaschke product $b$ without multiple roots. These subspaces are soTdense in the lattice of all invariant subspaces because the Blaschke products are soT-dense (and even norm dense) in the set of inner functions.

For $n \geqslant 2$, the eigenvectors of $\mathfrak{Q}_{n}^{*}$ turn out to be symmetric, meaning that the coefficients for $\xi_{w}$ depend only on the evaluation of $w$ on commuting variables. So they cannot be sufficient to determine the whole invariant subspace lattice. Indeed, they are also eigenvectors for $\mathfrak{R}_{n}^{*}$. Nevertheless, they will yield an important class of invariant subspaces. Let $\mathbb{B}_{n}$ denote the unit ball in $n$-dimensional Hilbert space. This makes sense even for $n=\infty$.

We note that eigenvectors of $\mathfrak{R}_{n}^{*}$ are also discussed in Example 8 of [1].

THEOREM 2.6. The eigenvectors for $\mathfrak{Q}_{n}^{*}$ are the vectors

$$
\nu_{\lambda}=\left(1-\|\lambda\|^{2}\right)^{1 / 2} \sum_{w \in \mathscr{F}_{n}} \overline{w(\lambda)} \xi_{w}=\left(1-\|\lambda\|^{2}\right)^{1 / 2}\left(I-\sum_{i=1}^{n} \overline{\lambda_{i}} L_{i}\right)^{-1} \xi_{1}
$$

for $\lambda$ in the unit ball $\mathbb{B}_{n}$. They satisfy

$$
L_{i}^{*} \nu_{\lambda}=\overline{\lambda_{i}} \nu_{\lambda}
$$

and $\left(p(L) \nu_{\lambda}, \nu_{\lambda}\right)=p(\lambda)$ for every polynomial $p=\sum_{w} a_{w} w$ in the semigroup algebra $\mathbb{C} \mathscr{F}_{n}$. This extends to the map $\varphi_{\lambda}(A)=\left(A \nu_{\lambda}, \nu_{\lambda}\right)$, which is a WOTcontinuous multiplicative linear functional on $\mathfrak{L}_{n}$. The vector $\nu_{\lambda}$ is cyclic for $\mathfrak{L}_{n}$. The subspace $\mathscr{M}_{\lambda}=\left\{\nu_{\lambda}\right\}^{\perp}$ is $\mathfrak{I}_{n}$-invariant, and its wandering subspace $\mathscr{W}_{\lambda}$ is $n$ dimensional, spanned by

$$
\zeta_{\lambda, i}=\lambda_{i} \xi_{1}-\left(1-\|\lambda\|^{2}\right)^{1 / 2} L_{i} \nu_{\lambda} \quad \text { for } 1 \leqslant i \leqslant n
$$


Proof. First note that if $\lambda$ is in $\mathbb{B}_{n}$, then

$$
\begin{aligned}
\sum_{w}|w(\lambda)|^{2} & =\sum_{k \geqslant 0} \sum_{|w|=k}|w(\lambda)|^{2} \\
& =\sum_{k \geqslant 0} \sum_{s_{i} \geqslant 0, s_{1}+\ldots+s_{n}=k} \frac{k !}{s_{1} ! \ldots s_{n} !}\left|\lambda_{1}\right|^{2 s_{1}} \ldots\left|\lambda_{n}\right|^{2 s_{n}} \\
& =\sum_{k \geqslant 0}\left(\sum_{i=1}^{n}\left|\lambda_{i}\right|^{2}\right)^{k}=\left(1-\|\lambda\|^{2}\right)^{-1}<\infty
\end{aligned}
$$

So $\nu_{\lambda}$ is defined for $\lambda$ in $\mathbb{B}_{n}$. Also note that

$$
\left\|\sum_{i=1}^{n} \overline{\lambda_{i}} L_{i}\right\|^{2}=\sum_{i=1}^{n}\left|\lambda_{i}\right|^{2}=\|\lambda\|^{2}<1 .
$$

So $I-\sum_{i=1}^{n} \overline{\lambda_{i}} L_{i}$ is invertible, and its inverse is given by the power series

$$
\left(I-\sum_{i=1}^{n} \overline{\lambda_{i}} L_{i}\right)^{-1}=\sum_{k \geqslant 0}\left(\sum_{i=1}^{n} \overline{\lambda_{i}} L_{i}\right)^{k}=\sum_{w \in \mathscr{F}} \overline{w(\lambda)} L_{w} .
$$

Thus the second identity for $\nu_{\lambda}$ is evident. Since

$$
\left(I-\sum_{i=1}^{n} \overline{\lambda_{i}} L_{i}\right) \nu_{\lambda}=\left(1-\|\lambda\|^{2}\right)^{1 / 2} \xi_{1},
$$

it is evident that $\nu_{\lambda}$ is cyclic.

A straightforward computation shows that

$$
\begin{aligned}
L_{i}^{*} \nu_{\lambda} & =\left(1-\|\lambda\|^{2}\right)^{1 / 2} L_{i}^{*} \sum_{w} \overline{\left(z_{i} w\right)(\lambda)} \xi_{z_{i} w} \\
& =\left(1-\|\lambda\|^{2}\right)^{1 / 2} \overline{\lambda_{i}} \sum_{w} \overline{w(\lambda)} \xi_{w}=\overline{\lambda_{i}} \nu_{\lambda} .
\end{aligned}
$$

Thus it follows that

$$
\left(L_{w} \nu_{\lambda}, \nu_{\lambda}\right)=w(\lambda)\left\|\nu_{\lambda}\right\|^{2}=w(\lambda) .
$$

This extends to polynomials by linearity. Evidently $\varphi_{\lambda}$ is WOT-continuous. If $A$ and $B$ are in $\mathfrak{L}_{n}$, then the scalars $\alpha=\varphi_{\lambda}(A)$ and $\beta=\varphi_{\lambda}(B)$ are determined by the identities $A^{*} \nu_{\lambda}=\bar{\alpha} \nu_{\lambda}$ and $B^{*} \nu_{\lambda}=\bar{\beta} \nu_{\lambda}$. Hence

$$
\varphi_{\lambda}(A B)=\left(A B \nu_{\lambda}, \nu_{\lambda}\right)=\left(\nu_{\lambda}, B^{*} A^{*} \nu_{\lambda}\right)=\alpha \beta=\varphi_{\lambda}(A) \varphi_{\lambda}(B) .
$$

Therefore $\varphi_{\lambda}$ is multiplicative.

Conversely, if $\nu=\sum_{w} a_{w} \xi_{w}$ satisfies $L_{i}^{*} \nu=\overline{\lambda_{i}} \nu$, then reversing the calculation in the first paragraph shows that $a_{w}=\overline{w(\lambda)} a_{1}$. Since $\nu$ is a vector of finite norm, this forces the condition $\|\lambda\|<1$, and $\nu=a_{1} \nu_{\lambda}$.

Since $\mathbb{C} \nu_{\lambda}$ is invariant for $\mathfrak{L}_{n}^{*}$, it follows that $\mathscr{M}_{\lambda}=\left\{\nu_{\lambda}\right\}^{\perp}$ is invariant for $\mathfrak{L}_{n}$. Let $\mathscr{W}_{\lambda}=\mathscr{M}_{\lambda} \ominus \sum_{i=1}^{n} L_{i} \mathscr{M}_{\lambda}$ denote the corresponding wandering space. Notice that

$$
L_{i} \mathscr{M}_{\lambda}=L_{i} \mathscr{H}_{n} \ominus \mathbb{C} L_{i} \nu_{\lambda} .
$$

If $\zeta$ belongs to $\mathscr{W}_{\lambda}$, we may write $\zeta=\alpha \xi_{1}+\sum_{i=1}^{n} \zeta_{i}$ for $\zeta_{i}$ in $L_{i} \mathscr{H}_{n}$. Since $\zeta$ is orthogonal to $L_{i} \mathscr{M}_{\lambda}$, it follows that $\zeta_{i}$ is a multiple of $L_{i} \nu_{\lambda}$, say $\zeta_{i}=\alpha_{i} L_{i} \nu_{\lambda}$. And 
since $\zeta$ is orthogonal to

we deduce that

$$
\nu_{\lambda}=(1-\|\lambda\|)^{1 / 2} \xi_{1}+\sum_{i=1}^{n} \overline{\lambda_{i}} L_{i} \nu_{\lambda},
$$

$$
0=\left(\zeta, \nu_{\lambda}\right)=(1-\|\lambda\|)^{1 / 2} \alpha+\sum_{i=1}^{n} \lambda_{i} \alpha_{i} .
$$

This equation has an $n$-dimensional solution space spanned by the $n$ solutions given by $\alpha=\lambda_{i}, \alpha_{i}=-(1-\|\lambda\|)^{1 / 2}$ and $\alpha_{j}=0$ otherwise. These yield the vectors $\zeta_{\lambda, i}$.

We note that the $\nu_{\lambda}$ all lie in, and indeed span, the symmetric Fock space. So while the $\mathscr{M}_{\lambda}$ are invariant for $\mathfrak{Q}_{n}$ and $\mathfrak{R}_{n}$, there are many other operators leaving them invariant as well. In particular, they do not come close to determining the invariant subspace lattice of $\mathfrak{L}_{n}$.

Now we turn to the problem of showing that $\mathfrak{L}_{n}$ is hyper-reflexive. We begin with a folklore result that algebras of infinite multiplicity are hyper-reflexive. A wOT-closed algebra $\mathfrak{A}$ is said to have infinite multiplicity if it is unitarily equivalent to an algebra of the form $\mathfrak{B} \otimes I$ where $I$ is the identity operator on an infinite-dimensional space.

THEOREM 2.7. Every WOT-closed algebra of infinite multiplicity is hyperreflexive with distance constant at most 9.

Proof. The algebra $\mathfrak{U}=\mathfrak{B} \otimes I$ is contained in $\mathscr{B}(\mathscr{H}) \otimes I$ which is an AF von Neumann algebra and thus is hyper-reflexive [8] with distance constant at most 4. It is a result of Arveson [2] that for any weak-* continuous linear functional $f$ on $\mathscr{B}(\mathscr{H})$, there are vectors $\xi$ and $\zeta$ in $\mathscr{H} \otimes \mathscr{H}^{\prime}$ (where $\mathscr{H}^{\prime}$ is any separable Hilbert space) such that for all $T$ in $\mathscr{B}(\mathscr{H})$,

$$
f(T)=((T \otimes I) \xi, \zeta) \text { and }\|\xi\|\|\zeta\|=\|f\| .
$$

Therefore $\mathscr{B}(\mathscr{H}) \otimes I$ has property $\mathbb{A}_{1}$. So the result now follows from $[11,18]$.

REMARK 2.8. Probably the number 9 is quite crude. However, this constant can be greater than 1 even for von Neumann algebras of infinite multiplicity. The usual example of a von Neumann algebra with constant greater than 1 is the $3 \times 3$ diagonal subalgebra $\mathscr{D}_{3}$ of $\mathscr{M}_{3}$ with respect to a basis $e_{1}, e_{2}, e_{3}$, which has distance constant $\sqrt{3 / 2}$ (see [12]). In fact, $\mathscr{D}=\mathscr{D}_{3} \otimes I$ acting on $\mathbb{C}^{3} \otimes \mathscr{H}$ also has distance constant at least $\sqrt{3 / 2}$. To see this, let $T$ in $\mathscr{M}_{3}$ be the matrix

$$
T=\frac{1}{\sqrt{3}}\left[\begin{array}{rrr}
0 & 1 & -1 \\
-1 & 0 & 1 \\
1 & -1 & 0
\end{array}\right]
$$

which has the property that $\|T\|=\operatorname{dist}\left(T, \mathscr{D}_{3}\right)=1$ but $\beta_{\mathscr{L}_{3}}(T)=\sqrt{2 / 3}$, where $\mathscr{L}_{3}$ is the lattice of projections in $\mathscr{D}_{3}$. Let $E$ denote a rank 1 projection $E=\xi_{0} \xi_{0}^{*}$ in $\mathscr{B}(\mathscr{H})$. Consider $X=T \otimes E$. First notice that for any $D$ in $\mathscr{D}_{3}$,

$$
\begin{aligned}
1=\|T\| & \leqslant\|T-D\| \\
& =\|(I \otimes E)(X-D \otimes I)(I \otimes E)\| \\
& \leqslant\|X-(D \otimes I)\| .
\end{aligned}
$$

Thus $\operatorname{dist}(X, \mathscr{D})=\|X\|=1$. 
To compute $\beta(X)=\sup _{P \in \mathscr{L}}\left\|P^{\perp} X P\right\|$ as $P$ runs over $\mathscr{L}=\operatorname{Lat}(\mathscr{D})$, it suffices to consider $\left\|P^{\perp} X P x\right\|$ where $x$ is a unit vector in the range of $P$. So one may then replace $P$ by the projection $P_{x}$ onto the cyclic invariant subspace for $\mathscr{D}$ generated by $x$. Let

$$
x=\sum_{i=1}^{3} x_{i}\left(e_{i} \otimes \zeta_{i}\right)
$$

where $\zeta_{i}$ are unit vectors in $\mathscr{H}$. We may assume that $\left(\zeta_{i}, \xi_{0}\right) \geqslant 0$, and so can write

$$
\zeta_{i}=\cos \theta_{i} \xi_{0}+\sin \theta_{i} \xi_{i}
$$

where $\xi_{i}$ is a unit vector orthogonal to $\xi_{0}$. Then

$$
\mathscr{D} x=\sum_{i=1}^{3} \oplus \mathbb{C}\left(e_{i} \otimes \zeta_{i}\right)
$$

Let $f=\sum_{i=1}^{3} x_{i} \cos \theta_{i} e_{i}$. Then

$$
X x=(T f) \otimes \xi_{0} \quad \text { and } \quad\|X x\|^{2}=\|T f\|^{2}=\sum_{i=1}^{3}\left|\left(T f, e_{i}\right)\right|^{2} .
$$

Let us assume the convention that addition of indices is calculated modulo 3 . Then

$$
T f=\frac{1}{\sqrt{3}} \sum_{i=1}^{3}\left(x_{i+1} \cos \theta_{i+1}-x_{i-1} \cos \theta_{i-1}\right) e_{i} .
$$

Now compute

$$
\left\|P_{x} X x\right\|^{2}=\sum_{i=1}^{3}\left|\left(T f \otimes \xi_{0}, e_{i} \otimes \zeta_{i}\right)\right|^{2}=\sum_{i=1}^{3}\left|\left(T f, e_{i}\right)\right|^{2} \cos ^{2} \theta_{i} .
$$

To simplify notation, let us write $c_{i}=\cos \theta_{i}$ and $s_{i}=\sin \theta_{i}$. Hence

$$
\begin{aligned}
\left\|P_{x}^{\perp} X x\right\|^{2} & =\|X x\|^{2}-\left\|P_{x} X x\right\|^{2} \\
& =\sum_{i=1}^{3}\left|\left(T f, e_{i}\right)\right|^{2} s_{i}^{2} \\
& =\frac{1}{3} \sum_{i=1}^{3}\left|x_{i+1} c_{i+1}-x_{i-1} c_{i-1}\right|^{2} s_{i}^{2} \\
& =\frac{1}{3}\left(\sum_{i=1}^{3}\left(s_{i+1}^{2}+s_{i-1}^{2}\right) c_{i}^{2}\left|x_{i}\right|^{2}-2 \operatorname{Re} \sum_{i=1}^{3} s_{i}^{2} c_{i-1} c_{i+1} x_{i-1} \overline{x_{i+1}}\right) \\
& =(A x, x),
\end{aligned}
$$


where we now think of $x$ as $\left(\begin{array}{l}x_{1} \\ x_{2} \\ x_{3}\end{array}\right)$ and

$$
\begin{aligned}
A & =\frac{1}{3}\left[\begin{array}{ccc}
\left(s_{2}^{2}+s_{3}^{2}\right) c_{1}^{2} & -c_{1} c_{2} s_{3}^{2} & -c_{1} c_{3} s_{2}^{2} \\
-c_{1} c_{2} s_{3}^{2} & \left(s_{1}^{2}+s_{3}^{2}\right) c_{2}^{2} & -c_{2} c_{3} s_{1}^{2} \\
-c_{1} c_{3} s_{2}^{2} & -c_{2} c_{3} s_{1}^{2} & \left(s_{1}^{2}+s_{2}^{2}\right) c_{3}^{2}
\end{array}\right] \\
& =\frac{1}{3}\left[\begin{array}{lll}
c_{1} & 0 & 0 \\
0 & c_{2} & 0 \\
0 & 0 & c_{3}
\end{array}\right]\left[\begin{array}{ccc}
s_{2}^{2}+s_{3}^{2} & -s_{3}^{2} & -s_{2}^{2} \\
-s_{3}^{2} & s_{1}^{2}+s_{3}^{2} & -s_{1}^{2} \\
-s_{2}^{2} & -s_{1}^{2} & s_{1}^{2}+s_{2}^{2}
\end{array}\right]\left[\begin{array}{lll}
c_{1} & 0 & 0 \\
0 & c_{2} & 0 \\
0 & 0 & c_{3}
\end{array}\right] \\
& =\frac{1}{3} \operatorname{diag}\left\{c_{1}, c_{2}, c_{3}\right\}\left(\sum_{i=1}^{3} s_{i}^{2} g_{i} g_{i}^{*}\right) \operatorname{diag}\left\{c_{1}, c_{2}, c_{3}\right\} \\
& =\frac{1}{3} \sum_{i=1}^{3} s_{i}^{2} h_{i} h_{i}^{*},
\end{aligned}
$$

where $g_{i}=e_{i+1}-e_{i-1}$ and $h_{i}=c_{i+1} e_{i+1}-c_{i-1} e_{i-1}$. Thus $A$ is positive, and hence

$$
\|A\| \leqslant \operatorname{Tr}(A)=\frac{1}{3} \sum_{i=1}^{3} c_{i}^{2}\left(s_{i+1}^{2}+s_{i-1}^{2}\right) .
$$

A calculus computation shows that this achieves its maximum when one of the $c_{i}$ equals 0 and another equals 1 , which yields the value $2 / 3$. And, when $c_{1}=0$ and $c_{2}=c_{3}=1$, one achieves $\|A\|=2 / 3$. Therefore $\beta(X)=\sqrt{2 / 3}$. So the distance constant for $\mathscr{D}$ is at least $\sqrt{3 / 2}$, just as in the case for $\mathscr{D}_{3}$.

We can now prove the main result of this section. To prove hyper-reflexivity, we make use of the fact that there are natural invariant subspaces with infinitedimensional wandering space that almost fill up the whole space. Then we reach the vector $\xi_{1}$ by making use of the invariant subspaces arising from the eigenvectors of the adjoint. It seems to us that a proof just of reflexivity would require at least this much. Surprisingly, because of the availability of these subspaces of infinite multiplicity, the hyper-reflexivity result is much more accessible than in the case $n=1$. Because of the commutant theorem and the structure of invariant subspaces, we also obtain a norm estimate from the corresponding derivation on $\mathfrak{R}_{n}$.

We note here that Arias and Popescu prove the reflexivity of $\mathfrak{L}_{n}$. Since hyperreflexive algebras are reflexive, the following result subsumes Theorem 4.1 of [1].

THEOREM 2.9. The algebras $\mathfrak{L}_{n}$ are hyper-reflexive. Moreover, for all $T$ in $\mathscr{B}\left(\mathscr{H}_{n}\right)$,

$$
\frac{1}{51} \operatorname{dist}\left(T, \mathfrak{Q}_{n}\right) \leqslant \sup _{L \in \operatorname{Lat}\left(\mathfrak{I}_{n}\right)}\left\|L^{\perp} T L\right\| \leqslant\left\|\left.\delta_{T}\right|_{\mathfrak{R}_{n}}\right\| \leqslant 2 \operatorname{dist}\left(T, \mathfrak{Q}_{n}\right)
$$

Proof. The case $n=1$ is hyper-reflexive by [11] with constant less than 19 . So we assume that $n \geqslant 2$.

Fix $T$ in $\mathscr{B}\left(\mathscr{H}_{n}\right)$ and let $\beta=\beta_{\mathrm{Lat}\left(\mathfrak{I}_{n}\right)(T)}$. Notice that $\mathfrak{Q}_{n}$ is unitarily equivalent to 
the restriction of $\mathfrak{L}_{n}$ to any cyclic subspace. So the restriction of $\mathfrak{L}_{n}$ to an invariant subspace $\mathscr{M}$ with an infinite-dimensional wandering space has infinite multiplicity, and therefore is hyper-reflexive with distance constant 9 by Theorem 2.7. Moreover, since the invariant subspaces of the restriction algebra on $\mathscr{M}$ are merely the invariant subspaces of $\mathfrak{L}_{n}$ contained in $\mathscr{M}$, it is clear that the distance estimate,

$$
\sup \left\{\left\|P^{\perp}\left(\left.T\right|_{\mathscr{M}}\right) P\right\|: P \in \operatorname{Lat}\left(\left.\mathfrak{Q}_{n}\right|_{\mathscr{M}}\right)\right\},
$$

for the restriction of $T$ to $\mathscr{M}$ is at most $\beta$.

Next we will show that there is an $A$ in $\mathfrak{L}_{n}$ such that

$$
\left\|\left.(T-A)\right|_{\left\{\xi_{1}\right\}^{\perp}}\right\| \leqslant 18 \sqrt{2} \beta .
$$

It will also follow that for any $\lambda$ in $\mathbb{B}_{n}$ and any wandering vector $\zeta$ for $\left\{\nu_{\lambda}\right\}^{\perp}$, there is a $B$ in $\mathfrak{Q}_{n}$ such that

$$
\left\|\left.(T-B)\right|_{\mathfrak{L}_{n}[\zeta]}\right\| \leqslant 9 \beta .
$$

Choose two subspaces $\mathscr{M}_{1}, \mathscr{M}_{2}$ in $\operatorname{Lat}\left(\mathfrak{Q}_{n}\right)$ with infinite-dimensional wandering spaces such that $\mathscr{M}_{1}+\mathscr{M}_{2}=\left\{\xi_{1}\right\}^{\perp}$ and the projections onto $\mathscr{M}_{i}$ commute. For example, let

$$
\mathscr{M}_{i}=\sum_{j \neq i} \sum_{k \geqslant 0}^{\oplus} \mathfrak{Q}_{n}\left[\xi_{z_{j} z_{i}^{k}}\right] .
$$

Also notice that with a minor modification, it can be arranged that any wandering vector for $\left\{\xi_{1}\right\}^{\perp}$ may be included in $\mathscr{M}_{1}$. By the preceding remarks, there are elements $A_{i}$ in $\mathfrak{Q}_{n}$ such that

$$
\left\|\left.\left(T-A_{i}\right)\right|_{\mathscr{M}_{i}}\right\| \leqslant 9 \beta \quad \text { for } i=1,2 .
$$

Notice that Theorem 2.1 implies that for any $L$ in $\operatorname{Lat}\left(\mathfrak{R}_{n}\right)$, the restriction mapping $\left.X \mapsto X\right|_{L}$ is isometric on $\mathfrak{L}_{n}$. Hence, as $\mathscr{M}_{1}$ and $\mathscr{M}_{2}$ have non-trivial intersection, we deduce that $\left\|A_{1}-A_{2}\right\| \leqslant 18 \beta$. Set $A=\frac{1}{2}\left(A_{1}+A_{2}\right)$, and note that $\left\|A-A_{i}\right\| \leqslant 9 \beta$. Since the projections onto $\mathscr{M}_{i}$ commute, given any vector $\eta$ in $\{\xi\}^{\perp}$, we may find orthogonal vectors $\eta_{i}$ in $\mathscr{M}_{i}$ such that $\eta=\eta_{1}+\eta_{2}$. Then by the CauchySchwarz inequality,

$$
\begin{aligned}
\left\|\left.(T-A)\right|_{\left\{\xi_{1}\right\}^{\perp}}\right\| & \leqslant \sup _{\eta \perp \xi_{1},\|\eta\|=1}\left\|(T-A) \eta_{1}\right\|+\left\|(T-A) \eta_{2}\right\| \\
& \leqslant\left(\left\|\left.(T-A)\right|_{\mathscr{M}_{1}}\right\|^{2}+\left\|\left.(T-A)\right|_{\mathscr{M}_{2}}\right\|^{2}\right)^{1 / 2} \leqslant 18 \sqrt{2} \beta .
\end{aligned}
$$

Now if $\lambda$ is in $\mathbb{B}_{n}$, Theorem 2.6 shows that $\left\{\nu_{\lambda}\right\}^{\perp}$ has an $n$-dimensional wandering space. Thus the restriction of $\mathfrak{L}_{n}$ to $\left\{\nu_{\lambda}\right\}^{\perp}$ is unitarily equivalent to its restriction to $\left\{\xi_{1}\right\}^{\perp}$. Therefore the above analysis applies equally well with $\nu_{\lambda}$ in place of $\xi_{1}$. In particular, given any wandering vector $\zeta$ for $\left\{\nu_{\lambda}\right\}^{\perp}$, there is a subspace $\mathscr{M}$ with infinite-dimensional wandering space containing $\zeta$. Thus, as above, there is a $B$ in $\mathfrak{Q}_{n}$ such that

$$
\left\|\left.(T-B)\right|_{\mathfrak{L}_{n}[\xi]}\right\| \leqslant\left\|\left.(T-B)\right|_{\mathscr{M}}\right\| \leqslant 9 \beta .
$$

Take $\lambda_{r}=(r, 0, \ldots, 0)$ for $0 \leqslant r<1$, set $\zeta_{r}=\zeta_{\lambda_{r}, 1} /\left\|\zeta_{\lambda_{r}, 1}\right\|$ and choose an element $B_{r}$ in $\mathfrak{Q}_{n}$ such that $\left\|\left.\left(T-B_{r}\right)\right|_{\mathfrak{I}_{n}\left[\xi_{r}\right]}\right\| \leqslant 9 \beta$. Then since $\mathfrak{Q}_{n}\left[\zeta_{r}\right]$ intersects $\left\{\xi_{1}\right\}^{\perp}$ in an infinite-dimensional subspace, we deduce again that $\left\|B_{r}-A\right\|<(9+18 \sqrt{2}) \beta$. 
Hence

$$
\left\|(T-A) \zeta_{r}\right\| \leqslant 18(1+\sqrt{2}) \beta
$$

But $\lim _{r \rightarrow 1} \zeta_{r}=\xi_{1}$, whence

$$
\left\|(T-A) \xi_{1}\right\| \leqslant 18(1+\sqrt{2}) \beta .
$$

Thus, using the Cauchy-Schwarz inequality again, we have

$$
\begin{aligned}
\|T-A\| & \leqslant\left(\left\|\left.(T-A)\right|_{\left\{\xi_{1}\right\}^{\perp}}\right\|^{2}+\left\|(T-A) \xi_{1}\right\|^{2}\right)^{1 / 2} \\
& <18 \sqrt{5+2 \sqrt{2}} \beta<51 \beta .
\end{aligned}
$$

Hence $\mathfrak{L}_{n}$ is hyper-reflexive.

Choose $\mathscr{M}$ in Lat $\left(\mathfrak{Q}_{n}\right)$ and a unit vector $\xi$ in $\mathscr{U}$ such that $\beta$ is approximated by $\left\|P_{\mathscr{M}}^{\perp} T \xi\right\|$. Without loss of generality, it may be supposed that $\mathscr{M}$ is the cyclic subspace generated by $\xi$. Hence there is a wandering vector $\zeta$ so that $\mathscr{M}=\mathfrak{Q}_{n}[\zeta]$. Therefore $P_{\mathscr{M}}=R_{\zeta} R_{\zeta}^{*}$ and

$$
\begin{aligned}
\left\|\left.\delta_{T}\right|_{\Re_{n}}\right\| & \geqslant\left\|T R_{\zeta}-R_{\zeta} T\right\| \geqslant\left\|P_{\mathscr{M}}^{\perp}\left(T R_{\zeta}-R_{\zeta} T\right) R_{\zeta}^{*}\right\| \\
& =\left\|P_{\mathscr{M}}^{\perp} T P_{\mathscr{M}}\right\| \geqslant\left\|P_{\mathscr{M}}^{\perp} T \xi\right\| .
\end{aligned}
$$

It follows that $\left\|\left.\delta_{T}\right|_{\Re_{n}}\right\| \geqslant \beta$, which establishes the second inequality.

The third inequality is standard. If $A$ is in $\mathfrak{Q}_{n}$ and $R$ belongs to $\mathfrak{R}_{n}$,

$$
\left\|\delta_{T}(R)\right\| \leqslant\|(T-A) R-R(T-A)\| \leqslant 2\|R\|\|T-A\| .
$$

Minimizing over $A$ in $\mathfrak{L}_{n}$ shows that $\left\|\left.\delta_{T}\right|_{\mathfrak{R}_{n}}\right\| \leqslant 2 \operatorname{dist}\left(T, \mathfrak{I}_{n}\right)$.

In the case $n=\infty$, the restriction to $\left\{\xi_{1}\right\}^{\perp}$ is already infinite multiplicity and thus has distance constant at most 9. So a review of the argument shows that the algebra $\mathfrak{L}_{\infty}$ has a distance constant of at most $18 \sqrt{2}$.

We also show that $\mathfrak{L}_{n}$ has the property $\mathbb{A}_{1}$, namely that every weak-* continuous linear functional $f$ on $\mathfrak{L}_{n}$ is given by a rank 1 operator acting as a functional on $\mathscr{B}(\mathscr{H})$. This means, in particular, that every weak-* continuous linear functional is WOT-continuous. Hence these two topologies coincide on $\mathfrak{I}_{n}$.

THEOREM 2.10. Suppose that $f$ is a weak-* continuous linear functional on $\mathfrak{I}_{n}$ for $n \geqslant 2$ with $\|f\|<1$. Then there are vectors $\xi$ and $\zeta$ with $\|\xi\|\|\zeta\|<1$ such that $f(A)=(A \xi, \zeta)$ for all $A$ in $\mathfrak{Q}_{n}$.

Proof. This follows immediately from the fact that there are countably many pairwise orthogonal cyclic subspaces $\mathfrak{Q}_{n}\left[\boldsymbol{\nu}_{k}\right]$ such that the restriction of $\mathfrak{Q}_{n}$ to each $\mathfrak{L}_{n}\left[\boldsymbol{\nu}_{k}\right]$ is canonically unitarily equivalent to $\mathfrak{Q}_{n}$. Given $f$ with $\|f\|<1$, there is (by the Hahn-Banach Theorem) a trace class operator $K$ with $\|K\|_{1}<1$ so that $f(A)=\operatorname{Tr}(A K)$. The singular decomposition of $K$ yields

$$
K=\sum_{k \geqslant 1} s_{k} \xi_{k} \zeta_{k}^{*}
$$

where $\xi_{k}$ and $\zeta_{k}$ are unit vectors and $s_{k} \geqslant 0$ such that $\sum_{k} s_{k}<1$. Let

$$
\xi:=\sum_{k} s_{k}^{1 / 2} R_{\nu_{k}} \xi_{k} \quad \text { and } \quad \zeta:=\sum_{k} s_{k}^{1 / 2} R_{\nu_{k}} \zeta_{k} \text {. }
$$


Then $\|\xi\|=\|\zeta\|<1$ and by part (iii) of Theorem 1.2,

$$
(A \xi, \zeta)=\sum_{k} s_{k}\left(A R_{\nu_{k}} \xi_{k}, R_{\nu_{k}} \zeta_{k}\right)=\sum_{k} s_{k}\left(A \xi_{k}, \zeta_{k}\right)=f(A)
$$

An immediate consequence of [11] or [18] for hyper-reflexivity is the following.

COROLlARY 2.11. Every WOT-closed unital subalgebra of $\mathfrak{L}_{n}$ is hyper-reflexive with constant at most 103.

Another immediate consequence that will prove to be important in $[\mathbf{1 3}]$ is as follows.

COROLlary 2.12. The weak-* and wot topologies on $\mathfrak{I}_{n}$ coincide.

\section{Atomic free semigroup algebras}

In this section, we will examine a more general class of isometric representations of the free semigroup. Say that an $n$-tuple of isometries $S=\left(S_{1}, \ldots, S_{n}\right)$ is free atomic if $\sum_{i=1}^{n} S_{i} S_{i}^{*} \leqslant I$ and there is an orthonormal basis $\left\{\xi_{k}\right\}$ for $\mathscr{H}$ for which there are endomorphisms $\pi_{i}: \mathbb{N} \rightarrow \mathbb{N}$ (where $1 \leqslant i \leqslant n$ ) and scalars $\lambda_{i, k} \in \mathbb{T}$ satisfying $S_{i} \xi_{k}=\lambda_{i, k} \xi_{\pi_{i}(k)}$. We call the corresponding representation of $\mathscr{F}_{n}$ atomic as well. Equivalently, this says that there is an atomic masa containing all the range projections $P_{w}=w(S) w(S)^{*}$. A free atomic semigroup algebra will be the wOT-closed algebra $\mathfrak{A}=\operatorname{Alg}\left(S_{1}, \ldots, S_{n}\right)$ generated by a set of free atomic isometries.

There is a connection between these representations and the permutation representations of $\mathcal{O}_{n}$ recently introduced and studied by Bratteli and Jorgensen in [5]. Permutation representations are a subclass of atomic representations: they are the same as above except that all scalars $\lambda_{i, k}=1$ and $\sum_{i=1}^{n} S_{i} S_{i}^{*}=I$. Bratteli and Jorgensen were interested in decomposing permutation representations into irreducible representations. However, the condition that $\lambda_{i, k}=1$ forced them to make certain restrictive assumptions. In general, to obtain a decomposition into irreducible representations, arbitrary scalars are needed as we shall see.

It turns out that atomic free semigroup algebras have a very nice structure theory. We show that the irreducible atomic representations of $\mathscr{F}_{n}$ can be completely classified up to unitary equivalence. This will then allow us to determine the invariant subspace structure. Every free atomic semigroup algebra will be shown to be hyper-reflexive.

Classifying all free semigroup algebras up to unitary equivalence is essentially equivalent to classifying all representations of the $\mathrm{C}^{*}$-algebra $\mathcal{O}_{n}$ up to unitary equivalence. However, this $\mathrm{C}^{*}$-algebra is $\mathrm{NGCR}$, meaning that it has no type $\mathrm{I}$ quotients. (Indeed, it is simple.) Such $\mathrm{C}^{*}$-algebras do not have a nice representation space in the sense [16] that there is no countable collection of Borel functions that distinguish the unitary invariants. Hence it is perhaps surprising that this class of atomic representations has such a nice classification.

There are three classes of atomic isometric representations of $\mathscr{F}_{n}$ which we will now describe.

(i) The left regular representation $\lambda$.

(ii) Let $x=z_{i_{1}} z_{i_{2}} \ldots z_{i_{m}} \ldots$ be an infinite word in the generators of $\mathscr{F}_{n}$; and define 
the sequence

$$
x_{m}=z_{i_{1}} z_{i_{2}} \ldots z_{i_{m}} \text { for } m \geqslant 0 .
$$

Let $\mathscr{F}_{n} x^{-1}$ denote the collection of words in the free group on $n$ generators of the form $v=u x_{m}^{-1}$ for $u$ in $\mathscr{F}_{n}$ and some $m \geqslant 0$. Identify words which are the same after cancellation, namely $u x_{m}^{-1}=\left(u x_{i_{m+1}}\right) x_{m+1}^{-1}$. Let $\mathscr{H}_{x}$ be the Hilbert space with orthonormal basis $\left\{\xi_{v}: v \in \mathscr{F}_{n} x^{-1}\right\}$. Define a representation $\pi_{x}$ of the free semigroup $\mathscr{F}_{n}$ on $\mathscr{H}_{x}$ by

$$
\pi_{x}\left(z_{i}\right) \xi_{v}=\xi_{z_{i} v} \quad \text { for } v \in \mathscr{F}_{n} x^{-1}
$$

Two words $x$ and $x^{\prime}=z_{j_{1}} z_{j_{2}} \ldots z_{j_{m}} \ldots$ are said to be tail equivalent if there are integers $k$ and $\ell$ so that $i_{m+k}=j_{m+\ell}$ for all $m \geqslant 0$. Let $[x]$ denote the tail equivalence class of $x$. When $x$ and $x^{\prime}$ are tail equivalent, there is a unitary operator $U: \mathscr{H}_{x} \rightarrow \mathscr{H}_{x^{\prime}}$ given by

$$
U \xi_{v x_{m+k}^{-1}}=\xi_{v x_{m+\ell}^{\prime-1}}^{\prime} \text { for } v \text { in } \mathscr{F}_{n} \text { and } m \geqslant 0 .
$$

It is easy to check that $U$ is a well-defined bijection between the bases which intertwines the two representations. Thus $U$ extends to a unitary operator which implements the unitary equivalence of the representations $\pi_{x}$ and $\pi_{x^{\prime}}$.

These representations are irreducible except when $[w]$ is periodic. The structure for the periodic case will be described later. Two representations $\pi_{x}$ and $\pi_{x^{\prime}}$ are unitarily equivalent if and only if $x$ and $x^{\prime}$ are tail equivalent.

(iii) Let $u=z_{i_{1}} \ldots z_{i_{k}}$ be a non-trivial word in $\mathscr{F}_{n}$ and let $\lambda$ be a scalar of modulus 1. The idea behind the following construction is to create a representation of $\mathscr{F}_{n}$ under which the word $u$ maps to an isometry which has $\lambda$ as an eigenvalue.

Let $\mathscr{K}_{u}$ be the Hilbert space with orthonormal basis,

$$
\left\{\xi_{s, w}: 1 \leqslant s \leqslant k \text { and } w \in \mathscr{F}_{n} \backslash \mathscr{F}_{n} z_{i_{s}}\right\} .
$$

Define a representation $\sigma_{u, \lambda}$ of $\mathscr{F}_{n}$ by

$$
\begin{array}{ll}
\sigma_{u, \lambda}\left(z_{i}\right) \xi_{s, 1}=\xi_{s-1,1} & \text { if } i=i_{s}, s>1, \\
\sigma_{u, \lambda}\left(z_{i}\right) \xi_{1,1}=\lambda \xi_{k, 1} & \text { if } i=i_{1}, \\
\sigma_{u, \lambda}\left(z_{i}\right) \xi_{s, 1}=\xi_{s, z_{i}} & \text { if } i \neq i_{s}, \\
\sigma_{u, \lambda}\left(z_{i}\right) \xi_{s, w}=\xi_{s, z_{i} w} & \text { if } w \neq 1 .
\end{array}
$$

Notice that $\sigma_{u, \lambda}(u) \xi_{k, 1}=\lambda \xi_{k, 1}$. The word $u$ will be called the central generator for this representation; and the sequence $\xi_{s, 1}$ for $1 \leqslant s \leqslant k$ of basis vectors which are cyclically permuted will be called the central cycle. Each basis vector $\xi_{s, 1}$ is mapped by $\sigma_{u, \lambda}\left(z_{i_{s}}\right)$ to the next vector in the cycle, $\xi_{s-1,1}$; but when $j \neq i_{s}$, $\sigma_{u, \lambda}\left(z_{j}\right) \xi_{s, 1}=\xi_{s, z_{j}}$. This is a wandering vector which sweeps out a copy of the left regular representation. So this representation consists of a central ring of $k$ nodes, and from each node, there are $n-1$ 'spokes' equivalent to the left regular representation.

It is evident that if the terms of $u$ are cyclically rotated, one obtains an equivalent representation. Let $\langle u\rangle$ denote the equivalence class of $u$ up to cyclic permutation.

The word $u$ is said to be primitive if it is not the power of a smaller word. It turns out that $\sigma_{u, \lambda}$ is irreducible precisely when $u$ is primitive. 
ExAmple 3.1. Let $\mathscr{H}=\ell^{2}(\mathbb{N})$. Define $S_{1} \xi_{n}=\xi_{2 n-1}$ and $S_{2} \xi_{n}=\xi_{2 n}$. Then $S_{1} \xi_{1}=\xi_{1}$. Clearly, $\xi_{1}$ is a cyclic vector for $\left(S_{1}, S_{2}\right)$. This is a representation of $\mathfrak{I}_{2}$ of type $\sigma_{z_{1}, 1}$.

ExAmple 3.2. Let $\mathscr{H}=\ell^{2}\left(\mathbb{Z} \times \mathbb{N}_{0}\right)$. Define two isometries in $\mathscr{B}(\mathscr{H})$ by $S_{1} \xi_{k, n}=\xi_{k, 2 n+1}, S_{2} \xi_{k, 0}=\xi_{k+1,0}$ and $S_{2} \xi_{k, n}=\xi_{k, 2 n}$ for $k \in \mathbb{Z}$ and $n>0$. In this case, every basis vector is a wandering vector. This example is a representation of $\mathfrak{l}_{2}$ equivalent to $\pi_{z_{2}^{\infty}}$.

ExAmple 3.3. Consider the $n=1$ case. The left regular representation is just the unilateral shift since $\mathbb{N}$ acts on $\ell_{2}(\mathbb{N})$ by translation. The only infinite word is $z_{1}^{\infty}$. This yields the shift on $\ell_{2}(\mathbb{Z})$, namely the bilateral shift. The only primitive word is $z_{1}$. The representations $\sigma_{z_{1}, \lambda}$ are the 1-dimensional representations as scalars. From the spectral representation of the bilateral shift as multiplication by $z$ on $L^{2}(\mathbb{T})$, it follows that $\pi_{z_{1}^{\infty}}$ is the direct integral of the $\sigma_{z_{1}, \lambda}$ with respect to Lebesgue measure. Finally, the general word is $z_{1}^{k}$. The representation $\sigma_{z_{1}^{k}, \lambda}$ is a cyclic weighted shift on $k$-dimensional space with all weights equal to 1 except one which is $\lambda$. This is unitary, and by the spectral theorem, it is diagonalizable with eigenvalues equal to the $k$ th roots of $\lambda$, say $\mu_{1}, \ldots, \mu_{k}$. Thus $\sigma_{z_{1, \lambda}^{k}} \simeq \sum_{i=1}^{\oplus k} \sigma_{z_{1}, \mu_{i}}$.

The main result of this section can now be stated.

THEOREM 3.4. Every representation of the free semigroup as a free atomic semigroup algebra is unitarily equivalent to a direct integral of representations which are each unitarily equivalent to one of

(i) the left regular representation $\lambda$,

(ii) $\pi_{x}$ corresponding to an aperiodic infinite word $x$ which is unique up to tail equivalence, or

(iii) $\sigma_{u, \lambda}$ for a primitive central generator $u$, which is unique up to a cyclical permutation, and a constant $\lambda$ in $\mathbb{T}$.

This decomposition is canonical.

We begin with an elementary lemma. For the rest of this section, let $\mathfrak{A}$ be an atomic free semigroup algebra $\operatorname{Alg}\left(S_{1}, \ldots, S_{n}\right)$, and let $\left\{\xi_{n}\right\}$ be a standard basis on which it acts.

LEMMA 3.5. If $\xi_{m}$ and $\xi_{n}$ are basis vectors, then either $\overline{\mathfrak{A} \xi_{m}}$ and $\overline{\mathfrak{A} \xi_{n}}$ are orthogonal or one contains the other, in which case there are a word $w$ and scalar $\lambda$ in $\mathbb{T}$ so that $w(S) \xi_{m}=\lambda \xi_{n}$ or vice versa.

Proof. If there is an intersection, it is spanned by standard basis vectors; and thus there are words $v$ and $w$ so that

$$
v(S) \xi_{m}=\lambda w(S) \xi_{n}
$$

If both $v$ and $w$ are non-trivial, they must have the same initial term because the ranges of distinct $S_{i}$ are orthogonal. Cancelling off terms from the left, we obtain a minimal pair $\left(v^{\prime}, w^{\prime}\right)$ such that $v^{\prime}(S) \xi_{m}=\lambda w^{\prime}(S) \xi_{n}$. It follows that one of $v^{\prime}$ or $w^{\prime}$ is equal to 1 . 
COROLlaRY 3.6. A standard basis vector $\xi$ is either a wandering vector for $\mathfrak{A}$ (so that $\left\{w(S) \xi: w \in \mathscr{F}_{n}\right\}$ is an orthonormal set), or there are a word $u \neq 1$ and scalar $\lambda$ in $\mathbb{T}$ such that $u(S) \xi=\lambda \xi$. The minimal choice of $u$ is unique, say $u=z_{i_{1}} \ldots z_{i_{k}}$. For any $j \neq i_{k}, S_{j} \xi$ is a wandering vector.

Proof. If $\xi$ is not a wandering vector, then the definition of atomic free semigroup algebra shows that there are distinct words $v$ and $w$ and a scalar $\lambda$ in $\mathbb{T}$ such that $v(S) \xi=\lambda w(S) \xi$. Apply the lemma and use cancellation to obtain a word $u=z_{i_{1}} \ldots z_{i_{k}}$ so that $u(S) \xi=\lambda \xi$. Moreover, if $u$ and $u^{\prime}$ are two words with this property, then one divides the other. Hence the minimal choice of the word $u$ is unique; and any other choice is a power of this minimal word.

Fix $j$ with $1 \leqslant j \leqslant k$ and set $\zeta=S_{j} \xi$. If $\zeta$ is not a wandering vector, then there are a scalar $\mu$ in $\mathbb{T}$ and a word $w$ so that $w(S) \zeta=\mu \zeta$. But then $\left(w z_{j}\right)(S) \xi=\mu S_{j} \xi$. Therefore, since the ranges of the $S_{i}$ are orthogonal, we find that $w=z_{j} w^{\prime}$ and so $\left(w^{\prime} z_{j}\right)(S) \xi=\mu \xi$. Hence $w^{\prime} z_{j}$ is a power of $u$. But this means that $j=i_{k}$.

Let $W=\left\{\xi_{n}: \xi_{n}\right.$ is wandering $\}$. Then $\mathscr{W}=\operatorname{span}(W)$ is invariant for $\mathfrak{A}$. This is because Corollary 3.6 shows that when $\xi_{n}$ belongs to $W$, then $w(S) \xi_{n}$ is also in $W$ for every word $w$ in $\mathscr{F}_{n}$.

LEMмA 3.7. For each primitive word $u$ and scalar $\lambda$ in $\mathbb{T}$, the projection $P_{u, \lambda}$ onto the subspace $\{\xi: u(S) \xi=\lambda \xi\}$ belongs to $\mathfrak{A}$.

Proof. We will show that

$$
P_{u, \lambda}=\underset{m \rightarrow \infty}{\text { sOT-lim}} p_{m}(\bar{\lambda} u(S))
$$

where $p_{m}(x)=(1 / m !) \sum_{j=1}^{m !} x^{m !+j}$. To verify convergence, it is enough to check each standard basis vector because the sequence is bounded. If $\xi$ is a standard basis vector such that $u^{k}(S) \xi$ is never a multiple of $\xi$, then by Corollary 3.6, $u^{k}(S) \xi$ is eventually a wandering vector. Hence large powers $u^{k}(S) \xi$ are pairwise orthogonal, and consequently their average $p_{m}(\bar{\lambda} u(S)) \xi$ converges to 0 in norm.

Next suppose $\xi$ is a unit vector such that $u(S)^{q} \xi=\mu \xi$ for some positive integer $q$ and scalar $\mu$ in $\mathbb{T}$. Assume $q$ is the least such integer, and let

$$
M=\operatorname{span}\left\{u(S)^{j} \xi: 1 \leqslant j \leqslant q\right\} .
$$

Clearly $M$ reduces $u(S)$. Let $\alpha_{j}$, for $1 \leqslant j \leqslant q$, be the $q$ th roots of $\mu$. The vectors

$$
y_{j}=\frac{1}{\sqrt{q}} \sum_{k=1}^{q} \bar{\alpha}_{j}^{k} u(S)^{k} \xi
$$

satisfy $u(S) y_{j}=\alpha_{j} y_{j}$ and are also an orthonormal basis for $M$. Hence $\left.u(S)\right|_{M}$ is diagonal with respect to this basis. Also $\sum_{j=1}^{q} y_{j}=\sqrt{q} \xi$.

Evidently, $p_{m}(1)=1$ for every $m \geqslant 1$, and

$$
p_{m}(x)=\frac{x}{1-x}\left[\frac{x^{m !}-\left(x^{m !}\right)^{2}}{m !}\right] \text { for } x \neq 1 \text {. }
$$

So $\lim p_{m}(\tau)=0$ for all $\tau$ in $\mathbb{T}$ with $\tau \neq 1$. Therefore,

$$
\lim _{m \rightarrow \infty} p_{m}(\bar{\lambda} u(S)) \xi= \begin{cases}0 & \text { if } \lambda^{q} \neq \mu, \\ \left(\xi, y_{j}\right) y_{j}=(1 / \sqrt{q}) y_{j} & \text { if } \lambda=\alpha_{j}, 1 \leqslant j \leqslant q .\end{cases}
$$

Hence $P_{u, \lambda}$ is defined in $\mathfrak{A}$ as a sot-limit. 
Next observe that when $u(S) \xi=\lambda \xi$, then $p_{m}(\bar{\lambda} u(S)) \xi=\xi$ for all $m$ and thus $P_{u, \lambda} \xi=\xi$. Conversely, if $P_{u, \lambda} \zeta=\xi$, then

$$
\begin{aligned}
u(S) \xi & =\lim _{m \rightarrow \infty} u(S) \frac{1}{m !} \sum_{t=1}^{m !}(\bar{\lambda} u(S))^{m !+t} \zeta \\
& =\lim _{m \rightarrow \infty} \lambda \frac{1}{m !} \sum_{t=1}^{m !}(\bar{\lambda} u(S))^{m !+t+1} \zeta \\
& =\lambda \xi+\lim _{m \rightarrow \infty} \frac{1}{m !}\left((\bar{\lambda} u(S))^{2 n !}-I\right) u(S) \zeta=\lambda \xi
\end{aligned}
$$

Thus $P_{u, \lambda}$ is a projection with the desired range.

COROLlary 3.8. If $u$ is a primitive word and $q$ is the least positive integer such that $u(S)^{q} \xi$ is a multiple $\mu \xi$ of $\xi$, then $\xi=\sum_{i=1}^{q} P_{u, \lambda_{i}} \xi$ where $\lambda_{i}$ are the $q$-th roots of $\mu$ for $1 \leqslant i \leqslant q$.

Proof. Equation (3) in the proof of Lemma 3.7 yields $P_{u, \lambda_{j}} \xi=(1 / \sqrt{q}) y_{j}$. Since $\xi=(1 / \sqrt{q}) \sum_{i=1}^{q} y_{j}$, the result follows.

Corollary 3.9. The projection P onto $\mathscr{W}^{\perp}$ lies in $\mathfrak{A}$.

Proof. We will show that $P$ equals $Q:=\sum P_{u, \lambda}$ as this sum runs over all primitive words $u$ and all scalars of modulus 1 . This sum is soT-convergent because the projections $P_{u, \lambda}$ are pairwise orthogonal projections; and hence $Q$ belongs to $\mathfrak{A}$. A basis vector $\xi$ is either wandering, or satisfies $v(S) \xi=\lambda \xi$ for some non-trivial word $v$ and scalar $\lambda$ in $\mathbb{T}$ by Corollary 3.6. Clearly, $Q \xi=0$ for every wandering vector $\xi$. In the latter case, Corollary 3.8 shows that $\xi$ lies in the range of $Q$. Hence $Q=P$ as claimed.

Now we can decide which of the atomic representations of types (i)-(iii) are irreducible, by which we mean that the image does not commute with any proper projection.

Proposition 3.10. Consider the representations of type (i)-(iii).

(i) The left regular representation $\lambda$ is irreducible.

(ii) The representation $\pi_{x}$ is irreducible except when $x$ is tail equivalent to a periodic word. In this case, there is a primitive word $u$ so that $x$ is equivalent to the infinite repetition of $u$. Then $\pi_{x}$ is unitarily equivalent to the direct integral $\int_{\mathbb{T}}^{\oplus} \sigma_{u, \lambda} d \lambda$ with respect to normalized Lebesgue measure.

(iii) The representation $\sigma_{u, \lambda}$ is irreducible when $u$ is primitive. When $u=v^{r}$ and $v$ is primitive, then $\sigma_{u} \simeq \sum_{j=1}^{\oplus r} \sigma_{v, \mu_{j}}$ where $\mu_{j}$ are the $r$-th roots of $\lambda$.

Proof. First consider the left regular representation $\lambda$. The rank 1 projection $\xi_{1} \xi_{1}^{*}=I-\sum_{i} S_{i} S_{i}^{*}$ lies in $C^{*}\left(\mathfrak{Q}_{n}\right)$. Thus if $Q$ is a reducing projection for $\mathfrak{Q}_{n}$, it must commute with $\xi_{1} \xi_{1}^{*}$. By replacing $Q$ by $Q^{\perp}$ if necessary, we may assume that $\xi_{1}$ lies in the range of $Q$. However, $\xi_{1}$ is cyclic and therefore $Q=I$. Hence $\lambda$ is irreducible.

Next consider the case (iii) representations $\sigma_{u, \lambda}$ when $u$ is primitive. The 
projection $P=P_{u, \lambda}$ is the rank 1 projection onto the span of $\xi_{k, 1}$, which is a cyclic vector. So as in case (i), this representation is irreducible.

When $u=v^{r}$ and $|v|=k$, decompose the space $\mathscr{K}_{u}$ as follows. Let $\mathscr{K}_{v}^{(r)}$ be the direct sum of $r$ copies of $\mathscr{K}_{v}$ with basis $\xi_{s, w}^{j}$ for $0 \leqslant j<r, 1 \leqslant s \leqslant k$ and $w$ in $\mathscr{F}_{n} \backslash \mathscr{F}_{n} z_{i_{s}}$. There is a unitary operator $W$ from $\mathscr{K}_{v}^{(r)}$ onto $\mathscr{K}_{u}$ given by $W \xi_{s, w}^{j}=$ $\xi_{s+k j, w}$. Consider $\sigma=W^{*} \sigma_{u, \lambda} W$. Then a calculation shows that

$$
\begin{array}{ll}
\sigma\left(z_{i}\right) \xi_{1,1}^{0}=\lambda \xi_{k, 1}^{r-1} & \text { if } i=i_{1}, \\
\sigma\left(z_{i}\right) \xi_{1,1}^{j}=\xi_{k, 1}^{j-1} & \text { if } i=i_{1} \text { and } j>0, \\
\sigma\left(z_{i}\right) \xi_{s, 1}^{j}=\xi_{s-1,1}^{j} & \text { if } i=i_{s} \text { and } s>1, \\
\sigma\left(z_{i}\right) \xi_{s, 1}^{j}=\xi_{s, z_{i}}^{j} & \text { if } i \neq i_{s}, \\
\sigma\left(z_{i}\right) \xi_{s, w}^{j}=\xi_{s, z_{i} w}^{j} & \text { if } w \neq 1 .
\end{array}
$$

Let $\mathscr{E}_{s, w}=\operatorname{span}\left\{\xi_{s, w}^{j}: 0 \leqslant j<r\right\}$ for $1 \leqslant s \leqslant k$ and $w \in \mathscr{F}_{n} \backslash \mathscr{F}_{n} z_{i_{s}}$. Thus we see that $\sigma(v)$ maps the subspace $\mathscr{E}_{1,1}$ onto itself via the unitary $U$ which is the weighted shift given by $U \xi_{1,1}^{j}=\xi_{1,1}^{j-1}$ for $2 \leqslant j \leqslant k$ and $U \xi_{1,1}^{1}=\lambda \xi_{1,1}^{r-1}$. The spectrum of $U$ is the set of $r$ th roots of $\lambda$, given by $\mu_{j}$ for $0 \leqslant j<r$.

Diagonalize $U$ with respect to a new basis $\zeta_{1,1}^{j}$ for $0 \leqslant j<r$ so that $U \zeta_{1,1}^{j}=\mu_{j} \zeta_{1,1}^{j}$. Then, using the fact that $\sigma\left(z_{i_{s}}\right) \mathscr{E}_{s, 1}=\mathscr{E}_{s-1,1}$ for $1<s \leqslant k$, define a basis $\zeta_{s, w}^{j}$ for $\mathscr{K}_{v}^{(r)}$ by setting $\zeta_{s-1,1}^{j}=\sigma\left(z_{i_{s}}\right) \zeta_{s, 1}^{j}$ for $0 \leqslant j<r$ and $1<s \leqslant k$; and let $\zeta_{s, w}^{j}=\sigma(w) \zeta_{s, 1}^{j}$ for $0 \leqslant j<r, 1 \leqslant s \leqslant k$ and $w$ in $\mathscr{F}_{n} \backslash \mathscr{F}_{n} z_{i_{s}}$. Then it follows that

$$
\sigma\left(z_{i_{1}}\right) \zeta_{1,1}^{j}=\mu_{j} \zeta_{k, 1}^{j} \text {. }
$$

Thus this exhibits a unitary equivalence between $\sigma$ and $\sum_{j=0}^{\oplus r-1} \sigma_{v, \mu_{j}}$.

Now turn to case (ii) when $[x]$ is aperiodic. Consider the standard basis vector $\xi_{1}$. We claim that the rank 1 projection $\xi_{1} \xi_{1}^{*}$ belongs to the von Neumann algebra $W^{*}\left(\pi_{x}\left(z_{1}\right), \ldots, \pi_{x}\left(z_{n}\right)\right)$. Indeed, $P_{m}=x_{m}(S) x_{m}(S)^{*}$ is the range projection onto the span of those basis vectors $\xi_{w}$ for $w$ in $x_{m} \mathscr{F}_{n} x^{-1}$. This is a decreasing sequence, and thus the sot-limit of the $P_{m}$ is a projection $P$. For each word $w$ and positive integer $k$, there is a unique word $w_{k}$ of length $k$ so that $\xi_{w}$ lies in the range of $w_{k}(S)$. This determines an infinite word which is easily seen to be $w x$, and $w_{k}$ consists of the first $k$ terms of this (reduced) word. Since $x$ is aperiodic, $w x$ and $x$ are different except for $w=1$. Hence for some sufficiently large $k$, $w_{k} \neq x_{k}$ and so $P_{k} \xi_{w}=0$. Therefore $P=\xi_{1} \xi_{1}^{*}$. This vector is clearly cyclic for $W^{*}\left(\pi_{x}\left(z_{1}\right), \ldots, \pi_{x}\left(z_{n}\right)\right)$, and hence $\pi_{x}$ is irreducible.

Finally, suppose that $x$ is periodic, being the infinite product of a primitive word $u=z_{i_{1}} \ldots z_{i_{k}}$. Denote a basis for $\mathscr{K}_{u} \otimes \ell_{2}(\mathbb{Z})$ by $\xi_{s, w}^{j}$ for $j$ in $\mathbb{Z}, 1 \leqslant s \leqslant k$ and $w$ in $\mathscr{F}_{n} \backslash \mathscr{F}_{n} z_{i_{s}}$. Define a unitary $W$ from this space onto $\mathscr{H}_{x}$ by

$$
W \xi_{s, w}^{j}=\xi_{w x_{s}^{-1} u^{j}} \quad \text { for } j \in \mathbb{Z}, 1 \leqslant s \leqslant k, w \in \mathscr{F}_{n} \backslash \mathscr{F}_{n} z_{i_{k-s}},
$$

where we adopt the convention that $x_{0}=1$ and $x_{-t}^{-1}=z_{i_{t}} \ldots z_{i_{1}}$ for $t \geqslant 1$. Then a calculation shows that $\sigma=W^{*} \pi_{x} W$ is given by

$$
\begin{array}{ll}
\sigma\left(z_{i}\right) \xi_{s, 1}^{j}=\xi_{s-1,1}^{j} & \text { if } i=i_{s}, s>1, \\
\sigma\left(z_{i}\right) \xi_{1,1}^{j}=\xi_{k, 1}^{j-1} & \text { if } i=i_{1}, \\
\sigma\left(z_{i}\right) \xi_{s, 1}^{j}=\xi_{s, z_{i}}^{j} & \text { if } i \neq i_{s}, \\
\sigma\left(z_{i}\right) \xi_{s, w}^{j}=\xi_{s, z_{i} w}^{j} & \text { if } w \neq 1 .
\end{array}
$$


The compression of $\sigma(u)$ to $\mathscr{E}_{1,1}=\operatorname{span}\left\{\xi_{1,1}^{j}: j \in \mathbb{Z}\right\}$ is the unitary bilateral shift $U \xi_{1,1}^{j}=\xi_{1,1}^{j-1}$. This operator is unitarily equivalent to multiplication by $z$ on $L^{2}(\mathbb{T})$. So as in the case (iii) above, we see that $\sigma$ decomposes as a direct integral of the $\sigma_{u, \lambda}$ over $\mathbb{T}$ with respect to Lebesgue measure.

Proof of Theorem 3.4. Decompose the space into minimal subspaces spanned by standard basis vectors which are reducing for $\mathfrak{A}$. The subspace containing a basis vector $\xi$ will contain its image under all words in the $S_{i}$ and $S_{j}^{*}$. However, because of the relation $(\mathrm{F})$, one need only consider words of the form $u(S) v(S)^{*}$. (Note that $v(S)^{*} \xi$ is either a non-zero multiple of another standard basis vector or it is 0.) For the rest of the argument, we may assume that the whole space is a minimal diagonally reducing subspace in this sense.

Let $W=\left\{\xi_{n}: \xi_{n}\right.$ is wandering $\}$. Then $\mathscr{W}=\operatorname{span}(W)$ is invariant for $\mathfrak{A}$. Suppose that $\mathscr{W}=\mathscr{H}$. Applying Lemma 3.5, we see that either $\mathfrak{A}$ has a cyclic wandering vector $\xi$, and so is unitarily equivalent to the left regular representation; or every $\xi$ in $W$ is a modulus 1 scalar multiple of the image of another member of $W$ under some $S_{i}$. Starting with any $\xi$ in $W$, we find that there is a unique $i_{1}$ so that $\xi$ belongs to $S_{i_{1}} \mathscr{H}$. Hence there is a unique sequence

$$
x_{m}=z_{i_{1}} z_{i_{2}} \ldots z_{i_{m}}
$$

for which there are a $\zeta_{m}$ in $W$ and scalar $\lambda_{m}$ so that $\xi=\lambda_{m} x_{m}(S) \zeta_{m}$. Let $x$ be the infinite word $x=z_{i_{1}} z_{i_{2}} \ldots z_{i_{m}} \ldots$. Given another element $\xi^{\prime}$ of $W$, let $x^{\prime}=$ $z_{j_{1}} z_{j_{2}} \ldots z_{j_{m}} \ldots$ be the infinite word determined by $\xi^{\prime}$. The diagonal irreducibility of $W$ means that there is a word $u v^{*}$ so that $\xi=u(S) v(S)^{*} \xi^{\prime}$. It is then evident that $u=x_{k}$ and $v=x_{\ell}^{\prime}$ for certain integers $k$ and $\ell$. Hence $\zeta=u(S)^{*} \xi=v(S)^{*} \xi^{\prime}$ is an element of $W$; and the two sequences $x$ and $x^{\prime}$ have the same tails $i_{m+k}=j_{m+\ell}$ for all $m \geqslant 0$. This equivalence class $[x]$ is therefore independent of the choice of basis vector.

For each word $w$ in $\mathscr{F}_{n} x^{-1}$, say $w=v x_{m}^{-1}$, define $\xi_{w}=v(S) x_{m}(S)^{*} \xi$. This is always a modulus 1 multiple of a standard basis vector. The diagonal irreducibility shows that every basis vector is achieved in this way. And because each basis vector is wandering, this map is also one-to-one. So we have a new basis for our space. With respect to this basis, it is evident that $u(S) \xi_{w}=\xi_{u w}$. So this shows that the representation is unitarily equivalent to $\pi_{x}$.

In fact, the class $[x]$ is a unitary invariant of the representation. To see this, consider a wandering vector $\zeta=\sum_{w} a_{w} \xi_{w}$ in $\mathscr{H}_{x}$ with the property that there is an infinite word $x^{\prime}$ so that for each $m \geqslant 1$, there is a vector $\zeta_{m}$ in $\mathscr{H}_{x}$ so that $x_{m}^{\prime}(S) \zeta_{m}=\zeta$. Then this infinite word coincides with the infinite word associated to any basis vector $\xi_{w}$ for which $a_{w} \neq 0$. Hence the class $[x]$ is determined intrinsically from the representation independent of the choice of basis; and thus is a unitary invariant.

Now suppose that $\mathscr{W} \neq \mathscr{H}$, and that $\mathscr{H}$ is diagonally irreducible. Then by Corollary 3.6, there are a minimal word $u=z_{i_{1}} \ldots z_{i_{k}} \neq 1$, a scalar $\lambda$ in $\mathbb{T}$ and a standard basis vector $\xi$ such that $u(S) \xi=\lambda \xi$. We will show that $\pi$ is unitarily equivalent to $\sigma_{u, \lambda}$. Let $\xi_{k, 1}=\xi$ and $\xi_{s-1,1}=S_{i_{s}} \xi_{s, 1}$ for $2 \leqslant s \leqslant k$. Note that $S_{i_{1}} \xi_{1,1}=\lambda \xi_{k, 1}$. Hence if $u_{s}=z_{i_{s+1}} \ldots z_{i_{k}} z_{i_{1}} \ldots z_{i_{s}}$ is the word obtained from $u$ by cyclical permutation of the letters, it follows that

$$
u_{s}(S) \xi_{s, 1}=\lambda \xi_{s, 1} \quad \text { for } 1 \leqslant s \leqslant k
$$


Now define $\xi_{s, w}=w(S) \xi_{s, 1}$ for all $w$ in $\mathscr{F}_{n} \backslash \mathscr{F}_{n} z_{i_{s}}$. Since $\xi_{s, w}=v(S) \xi$ for some word $v$ in $\mathscr{F}_{n}$, we see that $\xi_{s, w}$ is just a modulus 1 multiple of a standard basis vector. Also, note that by Corollary 3.6 and equation (4), each $\xi_{s, z_{i}}$ for $i \neq i_{s}$ is a wandering vector. Moreover, the set

$$
\left\{\xi_{s, w}: 1 \leqslant s \leqslant k, w \in \mathscr{F}_{n} \backslash \mathscr{F}_{n} z_{i_{s}}\right\}
$$

spans a diagonally irreducible subspace because it is mapped onto itself by the $n$ isometries $S_{i}$. Therefore it is a basis for $\mathscr{H}$. It is evident that this is unitarily equivalent to $\sigma_{\lambda, u}$.

It is clear that a cyclical permutation of $u$ does not change the unitary equivalence class of the representation. Suppose that $u=v^{s}$ where $v$ is primitive. Let $k=|v|$, and let $v_{i}$ denote the $k$ cyclic permutations of $v$; and let $\mu_{j}$ for $1 \leqslant j \leqslant s$ be the sth roots of $\lambda$. To see that $u$ up to permutation and $\lambda$ are unitary invariants, consider the space $\mathscr{W}^{\perp}$. This is the complement of the span of all wandering vectors in $\mathscr{H}$. By Lemma 3.7 and Corollary 3.8 , this subspace is the range of $P_{\langle u\rangle, \lambda}:=\sum_{i=1}^{k} \sum_{j=1}^{s} P_{v_{i}, \mu_{j}}$. Moreover, for any other primitive word $v^{\prime}$, the projection $P_{v^{\prime}, \mu}=0$; as is $P_{v_{i}, \mu}=0$ when $\mu^{s} \neq \lambda$. Hence the $v_{i}$ and $\mu_{j}$ are uniquely determined by the representation. Since $u_{i}=v_{i}^{s}$ are the cyclic permutations of $u$ and $\lambda=\mu_{i}^{k}$ where $k=\left|v_{i}\right|$ and $s$ is the number of roots, it follows that $\langle u\rangle$ and $\lambda$ can be recovered from the representation.

Proposition 3.10 shows that when $u$ is a higher power of a primitive word, then $\sigma_{u, \lambda}$ decomposes as the direct sum $\sum_{j=1}^{\oplus s} \sigma_{v, \mu_{j}}$ which are irreducible.

Finally, we show that this decomposition is canonical. The projection $P$ onto $\mathscr{W}^{\perp}$ can be used as above to determine the summands. For each primitive word $v$ and scalar $\lambda$ in $\mathbb{T}$, the multiplicity of the representation $\sigma_{v, \lambda}$ in an atomic representation $\sigma$ is given by the rank of $P_{v, \lambda}$. Of course, only one of the cyclic permutations of a primitive word should be used. The multiplicity of the left regular representation is given by the rank of $I-\sum_{i=1}^{n} S_{i} S_{i}^{*}$. If $x$ is an aperiodic

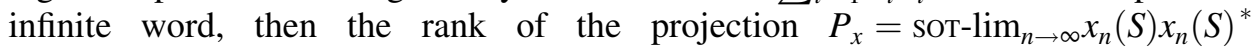
determines the multiplicity of $\pi_{x}$ (compare with the proof of Proposition 3.10). Finally, for each primitive word $u$, consider the periodic word $x=u^{\infty}$. The range projections $P_{n}=P^{\perp} u^{n}(S) u^{n}(S)^{*}$ decrease to the projection $P_{u}$ onto those vectors in $\mathscr{W}$ which are in the range of $u^{n}(S)$ for all $n \geqslant 1$. The multiplicity of $\pi_{x}$ equals the multiplicity of the bilateral shift $P_{u} u(S)$. By Proposition 3.10, $\pi_{x}$ decomposes uniquely as a direct integral of the irreducible representations $\sigma_{u, \lambda}$.

We collect the more precise information that came out in the proof as a corollary.

COROLlaRY 3.11. Every atomic representation $\rho$ of the free semigroup $\mathscr{F}_{n}$ may be decomposed uniquely as

$$
\rho \simeq \lambda^{(\alpha)} \oplus \sum^{\oplus} \pi_{[x]}^{\left(\beta_{[x]}\right)} \oplus \sum^{\oplus} \sigma_{\langle u\rangle, \lambda}^{\left(\gamma_{\langle u\rangle, \lambda}\right)}
$$

where $\alpha=\operatorname{rank}\left(I-\sum_{i=1}^{n} \rho\left(z_{i}\right) \rho\left(z_{i}\right)^{*}\right)$; for each infinite aperiodic word $x$ with tail equivalence class $[x]$,

$$
\beta_{[x]}=\operatorname{rank}\left(P_{x}\right) \quad \text { where } P_{x}=\underset{m \rightarrow \infty}{\operatorname{sOT}} \lim \rho\left(x_{m}\right) \rho\left(x_{m}\right)^{*} ;
$$

when $x$ is (equivalent to) a periodic word $u^{\infty}, \beta_{[x]}$ is the multiplicity of the bilateral shift $P_{x} \rho(u)$; and for each primitive word $u$ with cyclic permutation class 
$\langle u\rangle, \gamma_{\langle u\rangle, \lambda}=\operatorname{rank}\left(P_{u, \lambda}\right)$ where

$$
P_{u, \lambda}=\underset{m \rightarrow \infty}{\operatorname{sot}-\lim _{m}} p_{m}(\bar{\lambda} \rho(u)) \quad \text { and } \quad p_{m}(x)=\frac{1}{m !} \sum_{j=1}^{m !} x^{m !+j} .
$$

In order to understand the invariant subspace structure of free atomic algebras, we now study the algebra $P \mathfrak{A} P$ in detail. As above, $P$ is the projection onto the orthogonal complement of

$$
\mathscr{W}=\operatorname{span}\left\{\xi_{n}: \xi_{n} \text { is a wandering standard basis vector }\right\} .
$$

Lemma 3.12. Suppose that $u$ in $\mathscr{F}_{n}$ is a primitive word of length $k, \lambda$ is in $\mathbb{T}$ and let $\mathfrak{U}_{u, \lambda}$ be the WOT-closed algebra generated by the representation $\sigma_{u, \lambda}$ of $\mathscr{F}_{n}$. Then

$$
P \mathfrak{A}_{u, \lambda} P \simeq \mathscr{M}_{k} \quad \text { and } \quad \mathfrak{A}_{u, \lambda} P=\mathscr{B}\left(\mathscr{K}_{u}\right) P .
$$

In particular, a vector $\xi$ in $\mathscr{K}_{u}$ is cyclic for $\mathfrak{A}_{u, \lambda}$ if and only if $P \xi \neq 0$.

Proof. The projection $P_{u, \lambda}$ is the rank 1 projection onto the span of the basis vector $\xi_{k, 1}$. The operator $U=\sum_{i=1}^{n} P S_{i} P$ is a unitary on $P \mathscr{K}_{u}$ which cyclically shifts the basis vectors $\xi_{s, 1}$ for $1 \leqslant s \leqslant k$. Clearly these two operators generate $\mathscr{B}\left(P \mathscr{K}_{u}\right) \simeq \mathscr{M}_{k}$.

The vector $\xi_{1,1}$ is a cyclic vector for $\mathfrak{A}_{u, \lambda}$. Thus every vector $\xi$ such that $P \xi \neq 0$ is cyclic since the previous paragraph shows that $\xi_{1,1}(P \xi)^{*}$ belongs to $\mathfrak{A}_{u, \lambda}$. Finally, notice that if $\zeta$ is in $\mathscr{K}_{u}$ and $A_{n}$ is a sequence in $\mathfrak{A}_{u, \lambda}$ such that $\lim _{n \rightarrow \infty} A_{n} \xi_{1,1}=\zeta$, then

$$
\lim _{n \rightarrow \infty} A_{n}\left(\xi_{1,1} \xi_{s, 1}^{*}\right)=\zeta \xi_{s, 1}^{*}
$$

belongs to $\mathfrak{A}_{u, \lambda}$. Hence $\mathfrak{A}_{u, \lambda} P=\mathscr{B}\left(\mathscr{K}_{u}\right) P$.

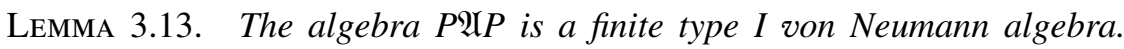

Proof. Clearly $P \mathfrak{A} P$ is wot-closed and is generated by $P S_{i} P$. To see that it self-adjoint, it suffices to show that their adjoints also lie in $P \mathfrak{A} P$. Consider a primitive word $u=z_{i_{1}} \ldots z_{i_{k}}$ and scalar $\lambda$ in $\mathbb{T}$. Given a non-zero vector $\eta$ in $P_{u, \lambda} \mathscr{H}$, an argument similar to the proof of Corollary 3.6 shows that if $S_{i} \eta$ is not a wandering vector, then $i=i_{k}$. Since $P=\sum P_{v, \mu}$ (where this soT-sum is taken over all primitive words $v$ in $\mathscr{F}_{n}$ and all $\mu$ in $\mathbb{T}$ ), we see that wandering vectors for $\mathfrak{A}$ belong to $\mathscr{W}$. Hence $S_{i} \eta$ belongs to $\mathscr{W}$ unless $i=i_{k}$. Since $\mathscr{W}$ is invariant for $\mathfrak{A}$ and $S_{i_{1}} \ldots S_{i_{k-1}}\left(S_{i_{k}} \eta\right)=\lambda \eta$, it follows that $P S_{i_{k}} \eta=S_{i_{k}} \eta$. Therefore,

$$
P S_{i} P_{u, \lambda}= \begin{cases}0 & \text { if } i \neq i_{k} \\ S_{i} P_{u, \lambda} & \text { if } i=i_{k}\end{cases}
$$

and hence $P S_{i} P_{u, \lambda}$ is a partial isometry. Then letting $u^{\prime}=z_{i_{1}} \ldots z_{i_{k-1}}$, one sees that

$$
\left(\bar{\lambda} P_{u, \lambda} u^{\prime}(S) P\right)\left(P S_{i} P_{u, \lambda}\right)=P_{u, \lambda} .
$$

Hence $\left(P S_{i} P_{u, \lambda}\right)^{*}=\bar{\lambda} P_{u, \lambda} u^{\prime}(S) P$ belongs to $\mathfrak{A}$. Since $P$ is the wot-convergent sum of the $P_{u, \lambda}$, it follows that $P S_{i}^{*} P$ belongs to $P \mathfrak{A} P$.

For each primitive word $u$, let $u_{s}$ for $1 \leqslant s \leqslant k$ be the cyclic permutations of $u$. The projection $P_{\langle u\rangle, \lambda}=\sum_{s=1}^{k} P_{u_{s}, \lambda}$ is invariant for $P \mathfrak{A} P$ by Theorem 3.4 because 
this is the projection onto the summand corresponding to multiples of $\sigma_{u, \lambda}$. Therefore it lies in the centre of $P \mathfrak{A} P$. By the previous lemma, $P_{\langle u\rangle, \lambda} \mathfrak{A} P P_{\langle u\rangle, \lambda} \simeq \mathscr{M}_{k}$. Since $P$ is the WOT-convergent sum of all the $P_{\langle u\rangle, \lambda}$, it follows that $P \mathfrak{A} P$ is the direct product of full matrix algebras.

We now are prepared to establish the second important result of this section.

THEOREM 3.14. Every atomic free semigroup algebra is hyper-reflexive with constant less than 51.

Proof. The left regular representation yields the algebra $\mathfrak{L}_{n}$ which is hyperreflexive with constant less than 51 by Theorem 2.9 .

The algebra $\mathfrak{A}_{[x]}$ determined by an infinite word $x$ acts on the space $\mathscr{H}_{x}$. This space is the increasing union of the cyclic subspaces $\mathfrak{I}\left[\xi_{x_{m}^{-1}}\right]$. As $\xi_{x_{m}^{-1}}$ is a wandering vector, the restriction to this subspace is unitarily equivalent to the left regular representation. On any subspace which is the direct sum of countably many pairwise orthogonal cyclic subspaces, the distance constant for the restriction is at most 9 by Theorem 2.7. In particular, inside $\mathfrak{A}\left[x_{m+1}^{-1}\right]$ there are countably many orthogonal cyclic subspaces including $\mathfrak{A}\left[x_{m}^{-1}\right]$ which are obtained exactly as in $\S 2$. Fix an operator $T$ in $\mathscr{B}\left(\mathscr{H}_{x}\right)$. For each $m \geqslant 1$, one finds an operator $A_{m}$ in $\mathfrak{U}$ so that

$$
\left\|\left.\left(T-A_{m}\right)\right|_{\mathfrak{Q}\left[x_{m}^{-1}\right]}\right\| \leqslant 9 \beta(T) .
$$

Thus an easy estimate shows that any wot-limit $A$ of a subsequence of $\left\{A_{m}\right\}$ satisfies $\|T-A\| \leqslant 9 \beta(T)$.

Now consider $\mathfrak{A}_{u, \lambda}$. By Lemma 3.12,

$$
\operatorname{dist}\left(T, \mathfrak{A}_{u, \lambda}\right)=\operatorname{dist}\left(T P^{\perp}, \mathfrak{A}_{u, \lambda} P^{\perp}\right) .
$$

The algebra $\mathfrak{A}_{u, \lambda} P^{\perp}$ is the direct sum of $k(n-1)$ copies of the left regular representation. Hence this algebra is hyper-reflexive with constant at most $18 \sqrt{2}$, as in the proof of Theorem 2.9.

Now look at the general case of an algebra $\mathfrak{A}$, which by Corollary 3.11 corresponds to a representation

$$
\rho \simeq \lambda^{(\alpha)} \oplus \sum^{\oplus} \pi_{[x]}^{\left(\beta_{[x]}\right)} \oplus \sum^{\oplus} \sigma_{\langle u\rangle, \lambda}^{\left(\gamma_{\langle u\rangle, \lambda}\right)}
$$

with two or more summands. Then $\mathfrak{A} P^{\perp} \mathscr{H}$ is spanned by wandering vectors. When there is no $\pi_{[x]}$, this space is determined by a wandering space of dimension at least 2 and has a distance constant at most $18 \sqrt{2}$. When there is at least one $\pi_{[x]}$, the space $P^{\perp} \mathscr{H}$ is the increasing union of such spaces with infinitedimensional wandering space. So it has distance constant at most 9 . The constant 9 is also valid if there are countably many summands in $\rho$.

So it remains to analyse $\mathfrak{A} P$. By Theorem 3.4 and Lemma 3.12, this space has the form

$$
\mathfrak{A} P \simeq \sum_{\langle u\rangle, \lambda}^{\oplus}\left(\mathscr{B}\left(\mathscr{K}_{u}\right) P_{\langle u\rangle, \lambda}\right)^{\left(\gamma_{\langle u\rangle, \lambda}\right)} .
$$

This space therefore equals $\mathfrak{M} P$ where $\mathfrak{W}$ is the type I von Neumann algebra

$$
\mathfrak{W}=\sum_{\langle u\rangle, \lambda}^{\oplus} \mathscr{B}\left(\mathscr{K}_{u}\right)^{\left(\gamma_{\langle u\rangle, \lambda}\right)} .
$$


As $P$ belongs to $\mathfrak{W}$ and $\mathfrak{W}$ has a distance constant at most 4 by Christensen's result [8], it follows that $\mathfrak{A} P$ also has distance constant at most 4 .

Combining these two results, we see that

$$
\operatorname{dist}(T, \mathfrak{A}) \leqslant\left(\operatorname{dist}(T P, \mathfrak{A} P)^{2}+\operatorname{dist}\left(T P^{\perp}, \mathfrak{A} P^{\perp}\right)^{2}\right)^{1 / 2} \leqslant \sqrt{664} \beta(T)<26 \beta(T) .
$$

This constant can be improved to $\sqrt{97}<10$ when the constant 9 can be used instead of $18 \sqrt{2}$.

EXAMPLE 3.15. This example is to show that a certain natural representation of $\mathscr{F}_{2}$ on $L^{2}(0,1)$ can be analysed using the structure of free atomic algebras. The natural analogue for all finite $n \geqslant 2$ is valid. Let $\mathscr{H}=L^{2}([0,1])$ equipped with Lebesgue measure and define isometries $S_{i}$ by

$$
\left(S_{1} f\right)(t)=\sqrt{2} f(2 t) \chi_{\left[0, \frac{1}{2}\right]}(t) \quad \text { and } \quad\left(S_{2} f\right)(t)=\sqrt{2} f(2 t-1) \chi_{\left[\frac{1}{2}, 1\right]}(t) .
$$

Then $S_{1} S_{1}^{*}+S_{2} S_{2}^{*}=I$. Let $\mathfrak{A}$ be the unital woT-closed algebra generated by $S_{1}$ and $S_{2}$.

Let $\eta_{0}=\chi_{[0,1]}, \quad \eta_{1}=\chi_{\left[0, \frac{1}{2}\right]}-\chi_{\left[\frac{1}{2}, 1\right]}$, and for every word $w \neq 1$ in $\mathscr{F}_{n}$, let $\eta_{w}=w(S) \eta_{1}$; note that these vectors are pairwise orthogonal since $\eta_{1}$ is a wandering vector for $\mathfrak{A}$.

Let $\mathscr{E}_{n}$ denote the span of $\left\{\eta_{0}, \eta_{w}:|w|<n\right\}$. Then it is easy to see that $\mathscr{E}_{n}$ equals the span of the characteristic functions of diadic intervals of length $2^{-n}$ and that this set is an orthonormal basis of $\mathscr{E}_{n}$. As the union of the $\mathscr{E}_{n}$ is dense in $L^{2}(0,1)$, altogether this yields an orthonormal basis of $L^{2}(0,1)$. In fact, this is the well-known Haar basis for $L^{2}(0,1)$. Therefore we see that $\eta_{1}$ is a wandering vector for $\mathfrak{A}\left[\eta_{1}\right]=\left\{\eta_{0}\right\}^{\perp}$. Since $\left(S_{1}-S_{2}\right) \eta_{0}=\sqrt{2} \eta_{1}$, it follows that $\eta_{0}$ is a cyclic vector for $\mathfrak{A}$.

Let $T_{1}=\left(S_{1}+S_{2}\right) / \sqrt{2}$ and $T_{2}=\left(S_{1}-S_{2}\right) / \sqrt{2}$. Then it is easy to verify that $T_{1}$ and $T_{2}$ are isometries with orthogonal ranges that generate the same algebra $\mathfrak{A}$. Moreover, $\eta_{1}$ is a wandering vector for this pair, and determines a basis $\zeta_{w}=w\left(T_{1}, T_{2}\right) \eta_{1}$, with $w \in \mathscr{F}_{2}$, for $\left\{\eta_{0}\right\}^{\perp}$. This basis consists of real functions of modulus 1; and for $|w|=n$, these functions are constant on diadic intervals of length $2^{-n-1}$. Moreover, $T_{1} \eta_{0}=\eta_{0}$ and $T_{2} \eta_{0}=\eta_{1}=\zeta_{1}$. So this pair determines a free atomic representation of $\mathscr{F}_{2}$. As it has a cyclic vector $\eta_{0}$ such that $T_{1} \eta_{0}=\eta_{0}$, this is evidently the representation $\sigma_{z_{1}}$ as in Example 3.1.

Let $U$ be the isometry of $\mathscr{H}_{2}$ onto $\left\{\eta_{0}\right\}^{\perp}$ given by $U \xi_{w}=\eta_{w}$. Then it follows that $\mathfrak{A}$ consists of all operators of the form $\eta \eta_{0}^{*}+U A U^{*}$ for $\eta$ in $L^{2}(0,1)$ and $A$ in $\mathfrak{L}_{2}$.

\section{References}

1. A. Arias and G. Popescu, 'Factorization and reflexivity on Fock spaces', Integral Equations Operator Theory 23 (1995) 268-286.

2. W. B. Arveson, 'Operator algebras and invariant subspaces', Ann. of Math. 100 (1974) 433-532.

3. W. B. Arveson, 'Interpolation problems in nest algebras', J. Funct. Anal. 20 (1975) 208-233.

4. A. Beurling, 'On two problems concerning linear transformations in Hilbert space', Acta Math. 81 (1949) 239-255.

5. O. Bratteli and P. JoRgensen, 'Iterated function systems and permutation representations of the Cuntz algebra', Mem. Amer. Math. Soc. to appear.

6. O. BRATTELI and D. RoBinson, Operator algebras and quantum statistical mechanics II (Springer, New York, 1981). 
7. J. Bunce, 'Models for $n$-tuples of non-commuting operators', J. Funct. Anal. 57 (1984) 21-30.

8. E. Christensen, 'Perturbations of operator algebras II', Indiana Univ. Math. J. 26 (1977) 891-904.

9. J. Cuntz, 'Simple C*-algebras generated by isometries', Comm. Math. Phys. 57 (1977) $173-185$

10. K. R. Davidson, 'On operators commuting with Toeplitz operators modulo the compact operators', J. Funct. Anal. 24 (1977) 291-302.

11. K. R. Davidson, 'The distance to the analytic Toeplitz operators', Illinois J. Math. 31 (1987) 265-273.

12. K. R. Davidson and M. Ordower, 'Some exact distance constants', Linear Algebra Appl. 208/209 (1994) 37-55.

13. K. R. DAvidson and D. Pitts, 'The algebraic structure of non-commutative analytic Toeplitz algebras', Math. Ann. 311 (1998) 275-303.

14. A. Frahzo, 'Models for non-commuting operators', J. Funct. Anal. 48 (1982) 1-11.

15. A. Frahzo, 'Complements to models for non-commuting operators', J. Funct. Anal. 59 (1984) 445-461.

16. J. Glimm, 'Type I C*-algebras', Ann. of Math. 73 (1961) 572-612.

17. J. Kraus and D. R. Larson, 'Some applications of a technique for constructing reflexive operator algebras', J. Operator Theory 13 (1985) 227-236.

18. J. Kraus and D. R. Larson, 'Reflexivity and distance formulae', Proc. London Math. Soc. (3) 53 (1986) 340-356.

19. G. POPESCU, 'Isometric dilations for infinite sequences of noncommuting operators', Trans. Amer. Math. Soc. 316 (1989) 523-536.

20. G. Popescu, 'Characteristic functions for infinite sequences of noncommuting operators', J. Operator Theory 22 (1989) 51-71.

21. G. Popescu, 'Multi-analytic operators and some factorization theorems', Indiana Univ. Math. J. 38 (1989) 693-710.

22. G. POPESCU, 'Von Neumann inequality for $\left(\mathscr{B}(\mathscr{H})^{n}\right)_{1}$ ', Math. Scand. 68 (1991) 292-304.

23. G. Popescu, 'Multi-analytic operators on Fock spaces', Math. Ann. 303 (1995) 31-46.

24. G. PopesCU, 'Functional calculus for noncommuting operators', Michigan Math. J. 42 (1995) $345-356$.

25. G. Popescu, 'Noncommuting disc algebras and their representations', Proc. Amer. Math. Soc. 124 (1996) 2137-2148.

26. M. ReED and B. Simon, Methods of mathematical physics, vol. II (Academic Press, New York, 1975).

27. S. Rosenoer, 'Distance estimates for von Neumann algebras', Proc. Amer. Math. Soc. 86 (1982) $248-252$.

28. D. SARAson, 'Algebras of functions on the unit circle', Bull. Amer. Math. Soc. 79 (1973) 286-299.

29. B. Sz. NAGY and C. FoIAŞ, Harmonic analysis of operators on Hilbert space (North Holland, London, 1970).

Department of Pure Mathematics

University of Waterloo

Waterloo

Ontario $N 2 L 3 G 1$

Canada

E-mail: krdavidson@math.uwaterloo.ca
Department of Mathematics

University of Nebraska

Lincoln

NE 68588

U.S.A.

E-mail: dpitts@math.unl.edu 